Delft University of Technology

\title{
Piloted simulator evaluation of a model-independent fault-tolerant flight control system
}

Tang, D; Pool, DM; Stroosma, O; Chu, QP; de Visser, CC

DOI

10.2514/6.2016-0085

Publication date

2016

Document Version

Accepted author manuscript

Published in

Proceedings of the AIAA guidance, navigation, and control conference

\section{Citation (APA)}

Tang, D., Pool, DM., Stroosma, O., Chu, QP., \& de Visser, CC. (2016). Piloted simulator evaluation of a model-independent fault-tolerant flight control system. In s.n. (Ed.), Proceedings of the AIAA guidance, navigation, and control conference (pp. 1-28). American Institute of Aeronautics and Astronautics Inc. (AIAA). https://doi.org/10.2514/6.2016-0085

Important note

To cite this publication, please use the final published version (if applicable).

Please check the document version above.

\section{Copyright}

Other than for strictly personal use, it is not permitted to download, forward or distribute the text or part of it, without the consent of the author(s) and/or copyright holder(s), unless the work is under an open content license such as Creative Commons.

Takedown policy

Please contact us and provide details if you believe this document breaches copyrights.

We will remove access to the work immediately and investigate your claim. 


\title{
Piloted Simulator Evaluation of a Model-Independent Fault-Tolerant Flight Control System
}

\author{
S. H. Tang* , D. M. Pool ${ }^{\dagger}$, O. Stroosma ${ }^{\ddagger}$, Q. P. Chu ${ }^{\S}$ and C. C. de Visser $₫$ \\ Delft University of Technology, Delft, 2600 GB, The Netherlands
}

\begin{abstract}
This paper for the first time evaluates the real-time performance of a sensor-based nonlinear dynamic inversion (SB-NDI) controller by means of a piloted proof-of-concept evaluation in a six degree of freedom research flight simulator. This fault-tolerant controller is completely model-independent and as a result does not require online identification. The inner loop of the controller incorporates an approximate dynamic inversion controller in combination with Lyapunov stability within a nonlinear dynamic inversion (NDI) framework and results in an adaptive model-free controller. The real-time performance, the fault-tolerant capabilities and the required physical workload of the controller are evaluated in the SIMONA research flight simulator with two flight scenarios consisting of approach procedures for the Flight1862 aircraft configuration and a vertical tail loss. The results are compared with full manual control and with an NDI attitude rate controller with a fixed aerodynamic model taken from literature. The results of this evaluation indicates that the designed multi-loop SB-NDI controller is capable of extending the survivability of the aircraft beyond what is possible with the baseline NDI controller. It also shows that the SB-NDI reduces pilot workload after a significant failure to levels comparable to the nominal aircraft.
\end{abstract}

\section{Nomenclature}

\begin{tabular}{|c|c|c|}
\hline 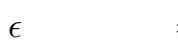 & $=$ & Time-scale separation value, a small positive number \\
\hline$J$ & $=$ & Moments of inertia matrix \\
\hline$b$ & $=$ & Wing span, $\mathrm{m}$ \\
\hline 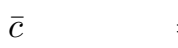 & $=$ & Mean aerodynamic chord, $\mathrm{m}$ \\
\hline$C$ & $=$ & Dimensionless coefficient \\
\hline$L, M, N$ & $=$ & Sum of aerodynamic and thrust moment around the $\mathrm{X}, \mathrm{Y}, \mathrm{Z}$ body axes, $\mathrm{Nm}^{2}$ \\
\hline$p, q, r$ & $=$ & Roll, pitch and yaw rate around the $\mathrm{X}, \mathrm{Y}, \mathrm{Z}$ body axes, rad/s \\
\hline$\omega$ & $=$ & Vector containing the angular rates $p, q, r$ \\
\hline$\delta_{a}, \delta_{e}$ & $=$ & Aileron, elevator deflection, deg \\
\hline$\delta_{r u}, \delta_{r l}$ & $=$ & Upper, lower rudder deflection, deg \\
\hline$P_{c}, P_{d}$ & $=$ & Collective, differential engine pressure ratio \\
\hline$P_{t}$ & $=$ & Total engine pressure ratio \\
\hline$V_{\text {tas }}$ & $=$ & True airspeed, $\mathrm{m} / \mathrm{s}$ \\
\hline$u, v, w$ & $=$ & Velocity along the $\mathrm{X}, \mathrm{Y}, \mathrm{Z}$ body axes, $\mathrm{m} / \mathrm{s}$ \\
\hline$a_{x}, a_{y}, a_{z}$ & $=$ & Specific forces along the X, Y, Z body axes, $\mathrm{m} / \mathrm{s}^{2}$ \\
\hline$\alpha, \beta$ & $=$ & Angle of attack, sideslip angle, rad \\
\hline$\phi, \theta, \psi$ & $=$ & Roll, pitch and heading angle, rad \\
\hline$h_{e}$ & $=$ & Height of the aircraft in the earth reference frame, $m$ \\
\hline sta & $=$ & Virtual input \\
\hline . & $=$ & Natural frequency, rad/s \\
\hline
\end{tabular}

* MSc Student, Faculty of Aerospace Engineering, Control and Simulation Division, 2626 HS Delft, The Netherlands.

${ }^{\dagger}$ Assistant Professor, Faculty of Aerospace Engineering, Control and Simulation Division, 2626 HS Delft, The Netherlands.

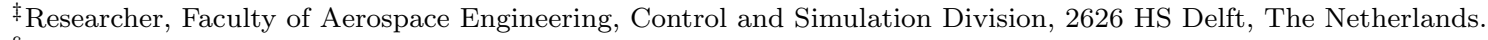

$\S$ Associate Professor, Faculty of Aerospace Engineering, Control and Simulation Division, 2626 HS Delft, The Netherlands.

『Assistant Professor, Faculty of Aerospace Engineering, Control and Simulation Division, 2626 HS Delft, The Netherlands. 


$\begin{array}{lll}\text { Subscript } & \\ \text { comm } & = & \text { Commanded } \\ \text { des } & = & \text { Desired } \\ \text { filt } & = & \text { Filtered } \\ \text { ref } & = & \text { Reference } \\ \text { il, ir, ol,or } & = & \text { Inner (wing) left, inner (wing) right, outer (wing) left, outer (wing) right }\end{array}$

\section{Introduction}

A worldwide civil aviation accident survey for the period of 2002 till 2011, conducted by the UK Civil Aviation Authority (CAA-UK) shows that in this time period nearly $40 \%$ of all the aircraft accidents involved loss of control. ${ }^{3}$ Some fatal examples of earlier cases are Flight123 in 1985, where the aircraft lost all hydraulics and lateral stability due to a vertical tail loss and Flight1862 that lost lost two engines and had to fly with reduced the actuator effectiveness. ${ }^{7,21}$ Despite the fatal outcomes, reconfigurable flight control might have been able to recover the aircraft by efficiently utilizing the remaining control inputs. ${ }^{19}$

Piloted simulations of one reconfigurable method, Adaptive Nonlinear Dynamic Inversion (ANDI), do not only allow a safe landing with a post-failure aircraft, but also reduce the workload of the pilot. ${ }^{15}$ However, the disadvantage of using adaptive control as a Fault-Tolerant Flight Controller (FTFC) is that they require a model identification algorithm for an aerodynamic model in order to compute the control actions. In order to further reduce the dependency on the internal aerodynamic model, incremental control methods can be used. $^{9,18}$ These methods only depend on the control effectiveness matrix to determine the control actions and therefore reduce the dependency of the aerodynamic model.

Although incremental control reduces the dependency of the aircraft model, it still requires an accurate estimate of the control effectiveness matrix to determine the control actions. Recent developments in the field of backstepping control reduces the model dependency of FTFC controllers even more, making a model-independent controller feasible. The proposed Sensor-Based Backstepping (SBB) controller of Falkena et al. integrated Hovakimyan's Approximate Dynamic Inversion (ADI) controller in the backstepping framework and is based on the singular perturbation theory. ${ }^{9,8,12,13}$ This SBB controller is fully model-independent and only uses the sign of the entries in the control input matrix to calculate the conventional control actions. As a result, the resulting controller is robust when changes occur to the controlled system which makes it a suitable FTFC implementation. Two studies already verified the fault-tolerant capabilities of the SBB controller by means of numerical simulations with a rudder runaway or with an engine separation. ${ }^{26,25}$

However, evaluations with human-in-the-loop simulations for the SBB controller are still limited in this field, even though they are important evaluations for a attitude rate control system. Literature only shows one example of a piloted simulation with the SBB controller in combination with a small aircraft. ${ }^{9}$ This study showed that a pilot is indeed capable of handling a small aircraft with a SBB flight controller. Although the simulation provided insight in the piloting characteristics, this study did not explicitly evaluate the fault-tolerant capabilities of the implemented SBB flight controller. Furthermore, evaluating a SBB flight controller with human-in-the-loop simulations does not only provide insight in the real-time performance and the pilot's physical workload of a SBB flight controller, but these piloted simulations also traverse various regions of the flight envelope in a natural way. As a result, these simulations provide more insight into how the controller performs when traversing through multiple regions of the flight envelopes, either with or without failures.

The objective of this paper is to provide an evaluation of the fault-tolerant capabilities and the real-time performance of a model-independent fault-tolerant attitude rate controller based on Hovakimyan et al.'s ADI controller by means of a piloted simulation in the SIMONA Research Simulator (SRS). The resulting attitude rate controller incorporates Hovakiman et al.'s ADI controller within the Nonlinear Dynamic Inversion (NDI) framework and uses Lyapunov stability as a definition for the virtual input. As a result, this paper will use the term Sensor-Based Nonlinear Dynamic Inversion (SB-NDI) to denote this combination.

The inner loop controlling the angular rates is based on the SB-NDI control framework and depends on the angular accelerations to compute the control inputs for the attitude control. These accelerations are provided by an approximate differentiator on the measurements of the angular rate sensors. Although 
angular accelerometers are available, these type of sensors are not widely available yet and thus the SB-NDI controller has to cope with gyro-differentiated rate-gyro measurements. Reference signals for the angular rates $p, q, r$ to control the attitude are provided by means of an NDI-based loop and contains a sideslip controller to prevent the aircraft from sideslipping. The sideslip angle is controlled by either the rudder or by differential thrust when the rudder is disabled due to a failure. Because of the two different designs of the SB-NDI controller, this paper assumes that a perfect Fault Detection and Identification (FDI) is present and successfully switches between the rudder or differential thrust for controlling the yaw rate.

The designed SB-NDI controller was implemented in the Reconfigurable Control for Vehicle Emergency Return (RECOVER) B747 100/200 model and evaluated with one pilot in the SRS. ${ }^{7,19}$ The real-time flight performance and the fault-tolerant capabilities of the multi-loop SB-NDI controller are evaluated with an approach procedure with a vertical tail loss and the Flight1862 failure scenarios. Besides evaluating the flight trajectories in a qualitative way, the physical workload of the pilot is analyzed by calculating the Root Mean Square (RMS) values of the applied deflections. The results of the multi-loop SB-NDI controller are compared with the flights results of the same scenarios with manual control. The multi-loop SB-NDI controller was also compared against an NDI-based attitude rate controller to investigate whether the SB-NDI controller requires a comparable physical control effort as the currently most widely used nonlinear control method. The NDI controller uses the same design and aerodynamic model as the work of Lombaerts et al. for the inner angular rate control loop. ${ }^{16}$ It should be explicitly stated that the flights mainly serve as a proof-of-concept flight and do not explicitly consider the detailed handling qualities of the flight controllers.

Section II discusses the mathematical framework of the SB-NDI controller. Subsequently, Section III describes the design of the fault-tolerant multi-loop SB-NDI attitude rate controller. Thereafter, Section IV discusses the used failure scenarios, the used flight scenarios and the evaluation setup for the real-time evaluation of the three flight controllers. This is followed by Section V which presents and analyzes the flight results. Finally, concluding remarks and recommendations for future research are given in Section VI.

\section{Sensor-Based Nonlinear Dynamic Inversion}

The Sensor-Based Nonlinear Dynamic Inversion (SB-NDI) controller is visualized by a block diagram in Figure 1. The SB-NDI is a novel controller that incorporates the Approximate Dynamic Inversion (ADI) controller of Hovakimyan et al. and the singular perturbation theory of Tikhonov within the Nonlinear Dynamic Inversion (NDI) control framework. ${ }^{12,13}$ The block diagram shows that the SB-NDI control law is dependent on the time derivative of the state vector which means that either accelerometers or a differentiation scheme should be available to obtain these derivatives. It should also be noted that the SB-NDI controller computes an incremental control input $\dot{\mathbf{u}}$ which needs to be integrated to obtain the total control input $\mathbf{u}$.

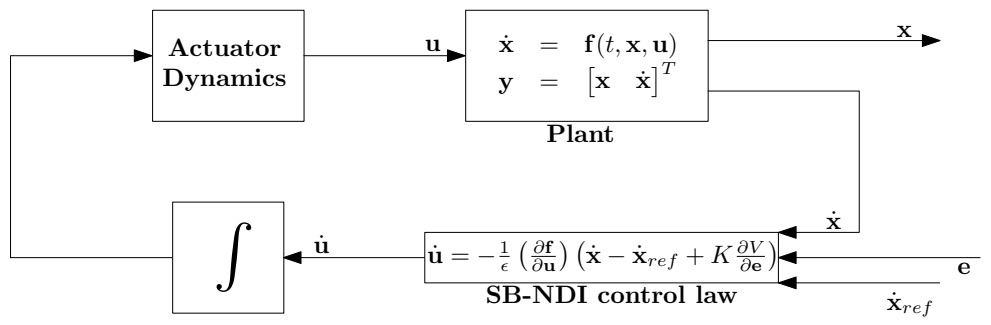

Figure 1: Block diagram of the SB-NDI control law.

In order to derive the SB-NDI control law for tracking a reference signal, consider a nonlinear, nonaffinein-control system $\mathbf{f}(t, \mathbf{e}, \mathbf{u})$ with the tracking error $\mathbf{e}$ defined as the difference between the state vector $\mathbf{x}$ and the reference signal $\mathbf{x}_{r e f}$. The ADI controller of Hovakimyan et al. given by Eq. (1) stabilizes the error dynamics by introducing the time-scale separation parameter $\epsilon$ to approximate the dynamic inversion of the system. $^{12}$ This approximation results in a 'fast dynamics' description of the original system as and it is assumed that these 'fast dynamics' stabilize the original system as long as it is controllable, minimum phase and sufficiently time-scale separated. ${ }^{8,12}$ 


$$
\epsilon \dot{\mathbf{u}}=-\operatorname{sign}\left(\frac{\partial \mathbf{f}}{\partial \mathbf{u}}\right) \mathbf{f}(t, \mathbf{e}, \mathbf{u}) \quad 0 \leq \epsilon \ll 1
$$

with:

$$
\mathbf{e}=\mathbf{x}-\mathbf{x}_{r e f}
$$

The SB-NDI reference tracking controller incorporates this ADI controller in the NDI framework and starts with defining the $\mathbf{f}(\boldsymbol{t}, \mathbf{e}, \mathbf{u})$ as given by Eq. (3). ${ }^{9,8,12}$

$$
\mathbf{f}(t, \mathbf{e}, \mathbf{u})=\dot{\mathbf{x}}-\dot{\mathbf{x}}_{d e s}
$$

The desired dynamics, $\dot{\mathbf{x}}_{\text {des }}$ can then be determined by defining a suitable Control Lyapunov Function $(\mathrm{CLF}) V(\mathbf{e})$ which is a function of the tracking error given by Eq. (2). The time derivative of $V(\mathbf{e})$ can then be written as:

$$
\begin{aligned}
\dot{V}(\mathbf{e}) & =\frac{\partial V(\mathbf{e})}{\partial \mathbf{e}} \dot{\mathbf{e}} \\
& =\frac{\partial V(\mathbf{e})}{\partial \mathbf{e}}\left(\dot{\mathbf{x}}-\dot{\mathbf{x}}_{r e f}\right)
\end{aligned}
$$

By promoting $\dot{\mathbf{x}}$ to a control variable, the following control law for the desired dynamics $\dot{\mathbf{x}}_{\text {des }}$ can be defined: $:^{9,8}$

$$
\dot{\mathbf{x}}_{\text {des }}=\dot{\mathbf{x}}_{r e f}-K \frac{\partial V(\mathbf{e})}{\partial \mathbf{e}}
$$

The error dynamics only becomes a globally asymptotically stable system if and only if the time derivative of the CLF is smaller than $0 .^{4,14}$ The error dynamics are therefore guaranteed to be stable as long as the entries of the diagonal gain matrix $K$ of Eq. (5) are larger than 0. Consequently, Eq. (6) is obtained as the final control law for the SB-NDI reference tracking controller by substituting the result back in Eq. (3).

$$
\epsilon \dot{\mathbf{u}}=-\operatorname{sign}\left(\frac{\partial \mathbf{f}}{\partial \mathbf{u}}\right)\left(\dot{\mathbf{x}}-\dot{\mathbf{x}}_{r e f}+K \frac{\partial V(\mathbf{e})}{\partial \mathbf{e}}\right) \quad K>0 \quad 0 \leq \epsilon \ll 1
$$

Eq. (6) shows the advantage of a SB-NDI reference tracking controller when it is designed for a nonlinear, affine-in-control system $\dot{\mathbf{x}}=f(\mathbf{x})+h(\mathbf{x}) \mathbf{u}$. For this case, the SB-NDI controller only depends on the sign of the entries in the input matrix $h(\mathbf{x})$, if the reference signal is not a function of the input vector $\mathbf{u} .^{9,8}$

This means that a SB-NDI tracking controller possesses two advantages in terms of robustness for a nonlinear affine-in-control system. The first and major advantage of the controller is that it does not depend on the function $f(\mathbf{x})$, because it does not require knowledge about this function in order to compute the control input. Therefore, large changes or uncertainties of the state matrix will not influence the performance of the controller. The second advantage is that the controller is robust to changes in $h(\mathbf{x})$, as long as the signs of the entries in $h(\mathbf{x})$ do not change.

\section{Attitude Rate Control Design}

A multi-loop SB-NDI attitude rate controller is designed to evaluate the fault-tolerant capabilities during a vertical tail loss and the Flight1862 scenario with the Reconfigurable Control for Vehicle Emergency Return (RECOVER) benchmark model. ${ }^{7,20}$ This benchmark consists of a nonlinear, dynamic model of a Boeing 747-100/200 and contains several failure cases which focuses either on actuator or structural failures.

In order to compare the performance of the multi-loop SB-NDI attitude rate controller with a static NDI attitude rate controller, the NDI controller uses the same NDI loop structure to control the attitude rates as the designed multi-loop SB-NDI controller. 


\section{A. Control Allocation}

The RECOVER model contains a Fly-By-Wire control system which allows independent control of all actuators. ${ }^{7,20}$ The control inputs of this model are defined as:

The inboard ailerons $\delta_{a i l}, \delta_{a i r}$ and the outboard ailerons $\delta_{a o l}, \delta_{a o r}$

The inboard elevators $\delta_{e i l}, \delta_{e i r}$ and the outboard elevators $\delta_{e o l}, \delta_{e o r}$

The upper and lower rudders $\delta_{r u}$ and $\delta_{r l}$

The inboard and outboard flaps $\delta_{f i}$ and $\delta_{f o}$

The twelve spoilers $\delta_{s p 1}$ to $\delta_{s p 12}$

The horizontal stabilizer $\delta_{i h}$

The four Engine Pressure Ratio (EPR) settings for the four engines $P_{t_{1}}$ to $P_{t_{4}}$

Control allocation methods can be used to determine the distribution of the control signals to the remaining, functional actuators. ${ }^{27}$ This paper groups the actuators in seven distinct groups given by Eq. (7) and (8) to equally distribute the control signals to the actuators. ${ }^{1}$ It should be noted that most of the spoilers, except for spoilers 5 to 8 , assist the ailerons in providing the rolling moment.

$$
\mathbf{u}=\left[\begin{array}{lllllll}
\delta_{a} & \delta_{e} & \delta_{r} & \delta_{i h} & \delta_{\text {flaps }} & P_{t_{\text {left }}} & P_{t_{\text {right }}}
\end{array}\right]^{T}
$$

with:

$$
\begin{aligned}
& \delta_{a}=\left[\begin{array}{llllllll}
\delta_{a i r} & \delta_{a i l} & \delta_{a o r} & \delta_{a o l} & \delta_{s p 1}, \delta_{s p 4} & \delta_{s p 2}, \delta_{s p 3} & \delta_{s p 10}, \delta_{s p 11} & \delta_{s p 9}, \delta_{s p 12}
\end{array}\right] \\
& \delta_{e}=\left[\begin{array}{llll}
\delta_{\text {eir }} & \delta_{\text {eil }} & \delta_{\text {eor }} & \delta_{\text {eol }}
\end{array}\right] \\
& \delta_{r}=\left[\begin{array}{ll}
\delta_{r u} & \delta_{r l}
\end{array}\right] \\
& \delta_{\text {flaps }}=\left[\begin{array}{ll}
\delta_{f i} & \delta_{f o}
\end{array}\right] \\
& P_{t_{\text {left }}}=\left[\begin{array}{ll}
P_{t_{1}} & P_{t_{2}}
\end{array}\right] \\
& P_{t_{\text {right }}}=\left[\begin{array}{ll}
P_{t_{3}} & P_{t_{4}}
\end{array}\right]
\end{aligned}
$$

\section{B. Overall Control Architecture}

The design of the designed multi-loop SB-NDI attitude rate controller is based on the hybrid Sensor-Based Backstepping (SBB) controller of Sun et al. and uses a similar control architecture. ${ }^{25}$ The multi-loop SB-NDI controller is visualized in Figure 2. The inner loop uses the SB-NDI framework to control the body angular rates by means of the ailerons $\delta_{a}$, elevators $\delta_{e}$ and rudders $\delta_{r}$. In case the rudder is disabled due to a failure, the SB-NDI controller uses differential thrust to control the yaw rate instead. The differential thrust is complemented with the an auto-throttle consisting of a PID controller to maintain the reference true airspeed $V_{\text {tas }}$ ref .

An outer loop complements the SB-NDI body angular rate control loop to translate the attitude roll rate $\dot{\phi}$ and pitch rate $\dot{\theta}$ to the body reference angular rates $\left[\begin{array}{ll}p r & r\end{array}\right]_{r e f}^{T}$. This loop is based on the NDI framework to provide the same outer loop structure for the SB-NDI and the reference NDI controller. The outer loop is indicated by 'NDI' block in Figure 2a. Section C further elaborates the design of the attitude control loop.

Besides an outer loop, the multi-loop SB-NDI controller depends on the angular accelerations which are provided by means of differentiated gyro-measurements due to limited availability of angular accelerometers. In addition, the controller requires the time derivatives of the computed reference angular rates $[p q r]_{r e f}^{T}$ by the NDI outer loop. A modified second order low-pass filtered differentiator computes the time derivatives, while imposing limits on the input signal. Besides these differentiators, the integral action of the multi-loop SB-NDI controller is regulated by an anti-integral windup scheme which is indicated by 'AI' in Figure 2. Sections D and E discusses the implementation of these complementary systems in more detail. 


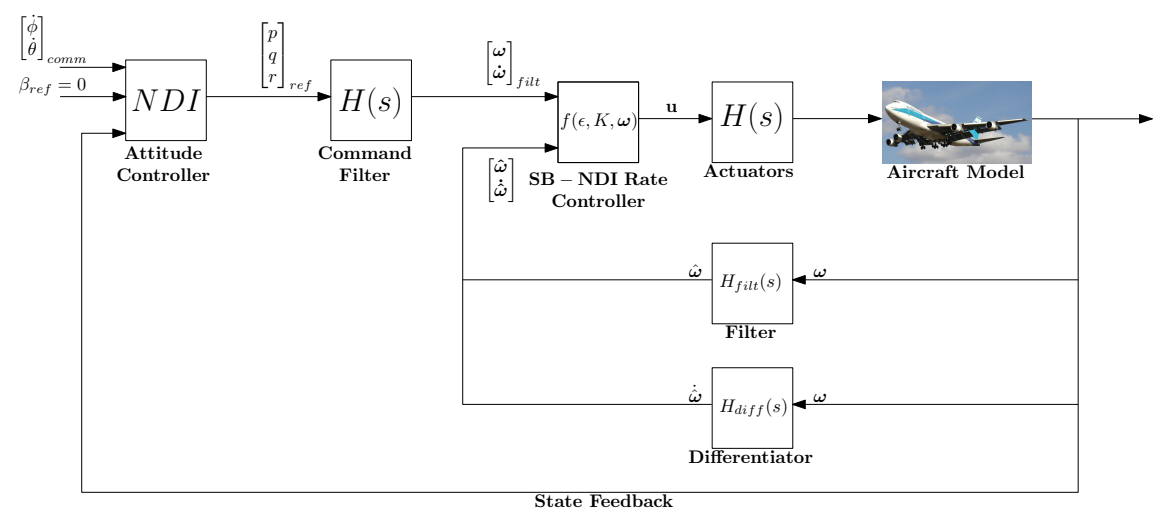

(a) Overall control design

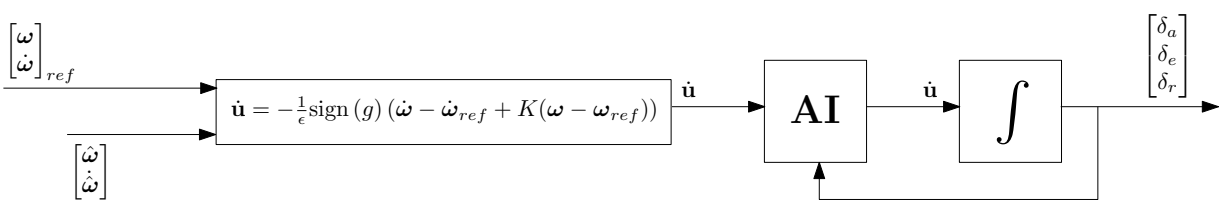

(b) Nominal SB-NDI rate controller. AI indicates the anti-integral windup scheme

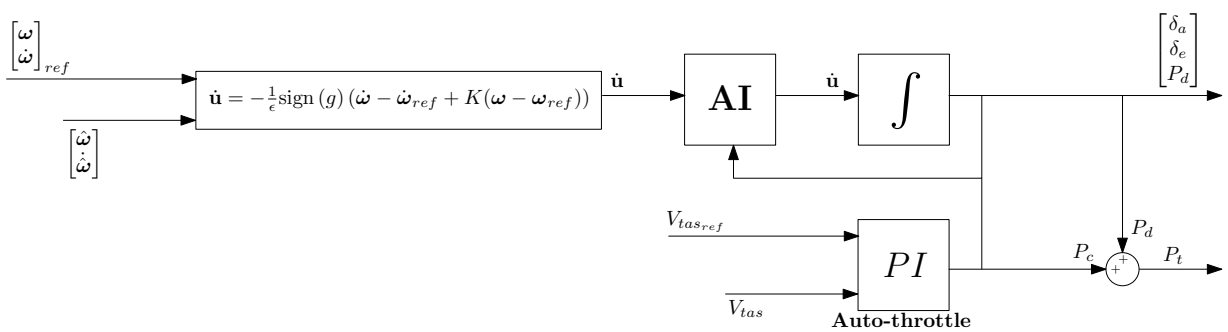

(c) SB-NDI rate controller with differential thrust. AI indicates the anti-integral windup scheme

Figure 2: Block diagrams of the SB-NDI controller design.

\section{Nominal Attitude Rate Control}

To derive a SB-NDI controller that controls the angular rates $\left[\begin{array}{ll}p r & r\end{array}\right]^{T}$ by using the aileron $\delta_{a}$, elevator $\delta_{e}$ and the rudder $\delta_{r}$, the moment equation is rewritten by assuming a linear relation between the moments generated by the control surfaces and the states. ${ }^{23}$ As a result, the moment equation around a trim point can be written as Eq. (9), with $\boldsymbol{\omega}$ being the angular rate vector $\left[\begin{array}{ll}p & r\end{array}\right]^{T}$ and $\mathbf{u}$ being the input vector $\left[\delta_{a} \delta_{e} \delta_{r}\right]^{T}$. This equation can be simplified to a simple linear in- and output relation as given by Eq. (10). Because this relation is an affine-in-control relation, the resulting SB-NDI controller will be fully independent of the state matrix $f(\boldsymbol{\omega})$ and will therefore only depend on the signs of the control effectiveness matrix $h$.

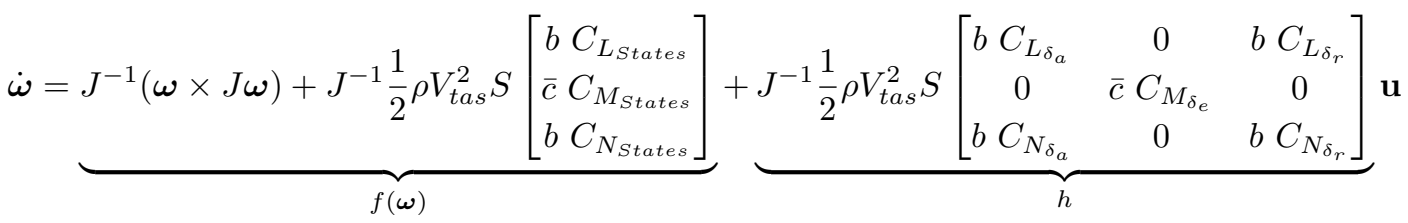

$$
\begin{aligned}
& =f(\boldsymbol{\omega})+h \mathbf{u}
\end{aligned}
$$

A quadratic CLF $V(\mathbf{e})=\frac{1}{2} \mathbf{e}^{2}$ with the tracking error $\mathbf{e}=\boldsymbol{\omega}-\boldsymbol{\omega}_{r e f}$ is used to determine the desired dynamics $\dot{\mathbf{x}}_{d e s}$ of the system. Its time derivative $\dot{V}(\mathbf{e})$ is then given by:

$$
\dot{V}(\mathbf{e})=\mathbf{e}\left(\dot{\boldsymbol{\omega}}-\dot{\boldsymbol{\omega}}_{r e f}\right)
$$

Eq. (12) is selected as the control law for the desired dynamics of the system $\dot{\boldsymbol{\omega}}_{\text {des }}$. Substituting Eq. (12) 
back into Eq. (11), it can be seen that the system only obtains global asymptotic stability if and only if the entries of the diagonal gain matrix $K$ are larger than 0 .

$$
\dot{\boldsymbol{\omega}}_{d e s}=-K \mathbf{e}+\dot{\boldsymbol{\omega}}_{r e f}
$$

with:

$$
K=\left[\begin{array}{ccc}
K_{p} & 0 & 0 \\
0 & K_{q} & 0 \\
0 & 0 & K_{r}
\end{array}\right]
$$

Substituting Eq. (12) into Eq. (6) results in the following control law for an angular rate controller:

$$
\dot{\mathbf{u}}=-\frac{1}{\epsilon} \operatorname{sign}(h)\left(\dot{\boldsymbol{\omega}}-\dot{\boldsymbol{\omega}}_{r e f}+K\left(\boldsymbol{\omega}-\boldsymbol{\omega}_{r e f}\right)\right) \quad K>0 \quad 0 \leq \epsilon \ll 1
$$

The control action $\mathbf{u}$ is obtained by integrating the result of Eq. (14) and its implementation is shown in Figure 2b with the gains listed in Table 1. The gains are based on simulations with perfect atmospheric conditions and without aircraft failures.

Furthermore, the sign of control effectiveness matrix $h$ is determined by two assumptions. For this paper, it is assumed that a fixed control effectiveness matrix at the trim point is available for $h$. A mass of $317,000 \mathrm{~kg}$,

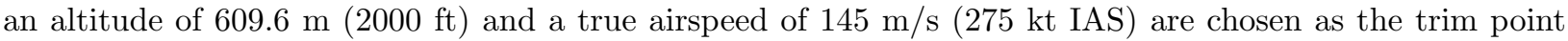
for the designed SB-NDI controller. It should be noted that this assumption may result in a suboptimal solution, especially when the entries are prone to sign changes or if the signs are inaccurately estimated. In addition, literature shows that it is sufficient to use the diagonal entries of $h$ for the computation of $\dot{\mathbf{u}}^{26,25}$ This paper uses the same approach which means that the roll, pitch and yaw rate are controlled by the ailerons, elevator and the rudder, respectively, without coupling.

Table 1: Rate control parameters for the SB-NDI controllers.

\begin{tabular}{cccc}
\hline \hline Parameter & Unit & SB-NDI & $\begin{array}{c}\text { SB-NDI } \\
\text { (Differential Thrust) }\end{array}$ \\
\hline$\epsilon$ & - & 0.25 & 0.35 \\
$K_{p}$ & $\frac{\mathrm{rad}}{\mathrm{rad} / \mathrm{s}}$ & 0.1 & 0.1 \\
$K_{q}$ & $\frac{\mathrm{rad}}{\mathrm{rad} / \mathrm{s}}$ & 0.2 & 0.2 \\
$K_{r}$ & $\frac{\mathrm{rad}}{\mathrm{rad} / \mathrm{s}}$ & 0.1 & 0.01 \\
\hline \hline
\end{tabular}

\section{Attitude Rate Control with Differential Thrust}

In the case where the rudder becomes unavailable due to, for example, a vertical tail loss or a rudder runaway, the control signals of the nominal SB-NDI flight controller cannot be used for the rudder. To compensate the yawing moment due to a rudder runaway or vertical tail loss, the engines can be used to provide differential thrust to generate a compensating yawing moment. ${ }^{22,6}$

However, the designed multi-loop SB-NDI still requires an Fault Detection and Identification (FDI) in order to switch between the two different implementations to control the sideslip angle. For the evaluation of the SB-NDI controller, it is assumed that such a FDI procedure is present and successfully switches to the SB-NDI controller controller with differential thrust when the rudder becomes unavailable. The SB-NDI controller with differential thrust starts with redefining the input vector $\mathbf{u}$ as:

$$
\mathbf{u}=\left[\begin{array}{lll}
\delta_{a} & \delta_{e} & P_{d}
\end{array}\right]^{T}
$$

The control law given by Eq. (12) remains the same and the only difference is that the control signal for the rudder is directed to the auto-throttle to provide a differential thrust. The total thrust for the engines is 
then given by Eq. (16) and (17) with $P_{c}$ being the collective thrust. ${ }^{22} P_{c}$ is defined as the computed thrust of the velocity control loop in order to maintain the reference true airspeed $V_{\text {tas }}$. . This controller consists of a PID controller with a proportional gain of $0.2 \frac{1}{\mathrm{~m} / \mathrm{s}}$ and an integral gain of $0.0025 \frac{1}{\mathrm{~s} \cdot \mathrm{m} / \mathrm{s}}$. The SB-NDI rate controller with the differential thrust and velocity control loop is visualized by Figure 2c. The gains of this controller were manually tuned and are listed in Table 1. The gains for the SB-NDI controller with differential thrust are based on flights with nominal flight conditions and with a vertical tail loss.

$$
\begin{aligned}
P_{t_{\text {left }}} & =P_{c}+P_{d} \\
P_{t_{\text {right }}} & =P_{c}-P_{d}
\end{aligned}
$$

\section{Attitude rate control loop}

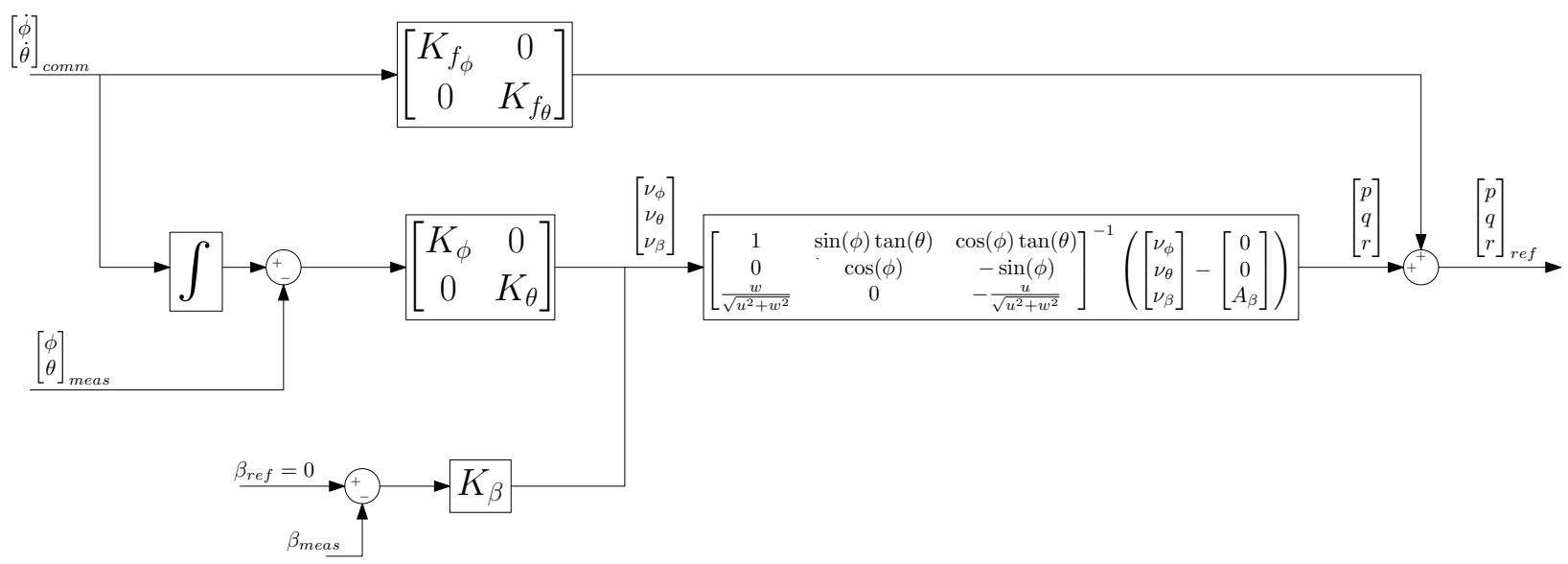

Figure 3: Block diagram of the NDI attitude rate control loop.

The attitude rate control loop is designed to control the attitude rates $\dot{\phi}$ and $\dot{\theta}$ by calculating the reference angular rate signal $\boldsymbol{\omega}$. The attitude rate control loop is based on the NDI framework and the inversion is given by Eq. (18). Besides the inversion, the NDI attitude rate control loop incorporates a feedforward term to improve the system response. Figure 3 visualizes the overall control loop structure of the attitude control loop.

$$
\left[\begin{array}{l}
p \\
q \\
r
\end{array}\right]=\left[\begin{array}{ccc}
1 & \sin (\phi) \tan (\theta) & \cos (\phi) \tan (\theta) \\
0 & \cos (\phi) & -\sin (\phi) \\
\frac{w}{\sqrt{u^{2}+w^{2}}} & 0 & -\frac{u}{\sqrt{u^{2}+w^{2}}}
\end{array}\right]^{-1}\left(\left[\begin{array}{c}
\nu_{\phi} \\
\nu_{\theta} \\
\nu_{\beta}
\end{array}\right]-\left[\begin{array}{c}
0 \\
0 \\
A_{\beta}
\end{array}\right]\right)
$$

with:

$$
\begin{aligned}
& {\left[\begin{array}{c}
\nu_{\phi} \\
\nu_{\theta}
\end{array}\right]=\left[\begin{array}{cc}
K_{\phi} & 0 \\
0 & K_{\theta}
\end{array}\right]\left(\int_{0}^{t}\left[\begin{array}{c}
\dot{\phi} \\
\dot{\theta}
\end{array}\right]_{\text {comm }} \mathrm{d} t-\left[\begin{array}{c}
\phi \\
\theta
\end{array}\right]_{\text {meas }}\right)} \\
& \nu_{\beta}=K_{\beta}\left(\beta_{\text {ref }}-\beta_{\text {meas }}\right) \\
& \beta_{\text {ref }}=0
\end{aligned}
$$

and:

$A_{\beta}=\frac{1}{\sqrt{u^{2}+w^{2}}}\left[-\frac{u v}{V_{\text {tas }}^{2}}\left(-g \sin \theta+a_{x}\right)+\left(1-\frac{v^{2}}{V_{\text {tas }}^{2}}\right)\left(g \sin \phi \cos \theta+a_{y}\right)-\frac{v w}{V_{\text {tas }}^{2}}\left(g \cos \phi \cos \theta+a_{z}\right)\right]$

where $\phi$ and $\theta$ are the Euler roll and pitch angle, $u, v, w$ the body velocities, $a_{x}, a_{y}, a_{z}$ the measurable specific forces along the body axes and $g$ is the gravitational acceleration. 
The same control loop structure is used to augment the static NDI attitude rate controller in order to translate the inputs $\dot{\phi}$ and $\dot{\theta}$ into the angular rates $[p q]_{r e f}^{T}$. The reference yaw rate for the NDI controller was already provided by a sideslip controller based on the work of Lombaerts et al. ${ }^{15}$

Table 2 displays the used gains for the SB-NDI and the NDI controller and were obtained by manually tuning the outer loop, such that both controllers have comparable control authority when compared with full manual control. The gains were tuned based on simulation flights with nominal flight conditions; aircraft failures were not taken into account, while tuning the attitude rate control loop.

It should also be noted that the outer loop also contains a sideslip controller to prevent the aircraft from sideslipping. Directional control is disabled for the pilots when using the SB-NDI and the NDI controller, meaning that the pedals cannot be used for yawing the aircraft. However, this choice also results in a constant 0 degrees reference signal for the reference sideslip angle $\beta_{\text {ref }}$, which means that the attitude rate control loop will try to prevent the aircraft from sideslipping.

Table 2: Attitude control parameters for the NDI outer loop.

\begin{tabular}{ccccc}
\hline \hline Parameter & Unit & SB-NDI & $\begin{array}{c}\text { SB-NDI } \\
\text { (Differential Thrust) }\end{array}$ & NDI \\
\hline$K_{f_{\phi}}$ & - & 0.8 & 0.8 & 0.25 \\
$K_{\phi}$ & $\frac{\mathrm{rad} / \mathrm{s}}{\mathrm{rad}}$ & 0.75 & 0.75 & 0.3 \\
$K_{f_{\theta}}$ & - & 0.8 & 0.8 & 0.2 \\
$K_{\theta}$ & $\frac{\mathrm{rad} / \mathrm{s}}{\mathrm{rad}}$ & 0.75 & 0.75 & 0.3 \\
$K_{\beta}$ & $\frac{\mathrm{rad} / \mathrm{s}}{\mathrm{rad}}$ & 1 & 0.1 & - \\
\hline \hline
\end{tabular}

\section{Differentiator and Command Filter Design}

Eq. (14) shows that the computation of the control inputs of the SB-NDI controller does not only depend on sensor feedback of the angular rates, but it also depends on a measurement of the angular accelerations in the current implementation. The angular accelerations are obtained by differentiating the rate gyromeasurements with a first-order low-pass differentiator with a cut-off frequency of $3 \mathrm{rad} / \mathrm{s}$. Although angular accelerations can be measured by using angular accelerometers, these sensors are not widely available yet and thus gyro-differentiation is used instead.

Besides the approximate differentiator, the angular rates from the angular rate sensors are filtered with a first-order low-pass filter with the same cut-off frequency as the differentiator. This filter is mainly used to compensate the introduced lag for the angular accelerations. The implementation of the angular rate sensor filter and the differentiator is shown in Figure 2a.

Furthermore, the SB-NDI controller is also dependent on the time derivatives of the reference rates generated by the pilot. To prevent the aircraft from leaving its safe flight envelope, a command filter based on the modified second-order differentiator of Farrell et al. was implemented as a measure. ${ }^{10}$ This differentiator computes the time derivatives, while limiting the input command rates by imposing magnitude limits on the input signal. The used values for the natural frequency $\omega_{n}$, the damping frequency $\zeta$ and magnitude limits of this filter are shown in Table 3 and are taken from the work of Sun et al. ${ }^{25}$

Table 3: Command filter parameters and ranges. ${ }^{25}$

\begin{tabular}{cccccc}
\hline \hline Command & Unit & Minimum & Maximum & $\omega_{n}$ & $\zeta$ \\
\hline$p$ & $\mathrm{rad} / \mathrm{s}$ & -0.2 & 0.2 & $6 \mathrm{rad} / \mathrm{s}$ & 1 \\
$q$ & $\mathrm{rad} / \mathrm{s}$ & -0.2 & 0.2 & $3 \mathrm{rad} / \mathrm{s}$ & 1 \\
$r$ & $\mathrm{rad} / \mathrm{s}$ & -0.1 & 0.1 & $3 \mathrm{rad} / \mathrm{s}$ & 1 \\
\hline \hline
\end{tabular}




\section{E. Integral windup}

Because the SB-NDI controller integrates the control signal $\dot{\mathbf{u}}$, integral windup problems may occur. ${ }^{9}$ This problem results in oversaturation of the actuators and can cause dangerous situations in case of an aircraft failure. The saturation effects are removed by applying conditional integration scheme that determines the integral action based on conditions when integration is allowed. ${ }^{5,29}$ The SB-NDI controller uses a conditional integration scheme that stops the integral action when the current integrated value reaches the maximum limit and if the sign of the integral action $\dot{\mathbf{u}}$ generated by the SB-NDI causes the actuator to saturate further. Integration only continues if $\dot{\mathbf{u}}$ moves the integral away from the integration limits or if the integral is not saturated.

The incorporation of the anti-integral windup scheme with the SB-NDI controller is indicated by 'AI' in Figures $2 \mathrm{~b}$ and $2 \mathrm{c}$. This scheme uses the lower limits of the actuator groups defined in Eq. (8) and are listed in Table $4 .^{7}$

Table 4: Limits for the conditional integration scheme. ${ }^{7}$

\begin{tabular}{cccc}
\hline \hline Actuator & Unit & Minimum & Maximum \\
\hline$\delta_{a}$ & Degrees & -20 & 20 \\
$\delta_{e}$ & Degrees & -23 & 17 \\
$\delta_{r}$ & Degrees & -25 & 25 \\
$P_{t}$ & - & 0 & 1.62 \\
\hline \hline
\end{tabular}

\section{Controller Evaluation Setup}

The designed multi-loop SB-NDI controller is evaluated in a piloted flight simulation in the SIMONA Research Simulator (SRS) with the full-motion capabilities of the simulator. The evaluation run consists of an approach procedure to evaluate the fault-tolerant capabilities of the SB-NDI controller during a vertical tail loss and the Flight1862 flight configuration without wind, gusts and turbulence. The landing itself is not part of the flight procedures, because an accurate ground effect and a landing gear model are not available in the RECOVER model.

The same flight plans are flown for the aircraft with full manual control and an NDI-based attitude rate controller with a static aerodynamic model to compare the performance of the multi-loop SB-NDI controller. ${ }^{16}$ The flights mainly serve as a proof-of-concept evaluation and therefore only provide a qualitative analysis of the flight results. Furthermore, changes in the physical workload of the pilot are also taken into account for the analysis. Detailed handling qualities are not explicitly evaluated by metrics such as CooperHarper ratings, because no performance criteria were defined for the flight maneuvers.

A retired B767 pilot with test flight experience was asked to fly the designed SB-NDI and the NDI attitude rate controllers for the proposed scenarios in a fixed sequence (no failure, engine separation, vertical tail loss) for every controller. The evaluation of each controller consisted of four flights: besides the three proposed scenarios, the pilot was also given one training flight to get accustomed with the controllers. There was no time limit imposed on the pilot when flying the approach maneuvers.

\section{A. Failure Scenarios}

The evaluation of the fault-tolerant capabilities of the SB-NDI controller focuses on two failure cases. As was stated in Section II, the SB-NDI controller is independent of the state matrix which makes the SB-NDI controller robust when failures introduce changes in this matrix. Therefore, the vertical tail loss scenario is the most representative scenario in assessing the fault-tolerant features of the designed SB-NDI controller.

Besides the vertical tail loss scenario, this paper also evaluates the fault-tolerant capabilities of the SB-NDI controller in combination with the Flight1862 failure configuration to illustrate the capabilities of the controller with a well-known and previously studied failure case. ${ }^{7,21,15}$ This configuration severely limits the safe flight envelope and reduces the actuator effectiveness. Figure 4 shows the overall effects of the engine separation on the aircraft for the Flight1862 case. $^{20}$ 


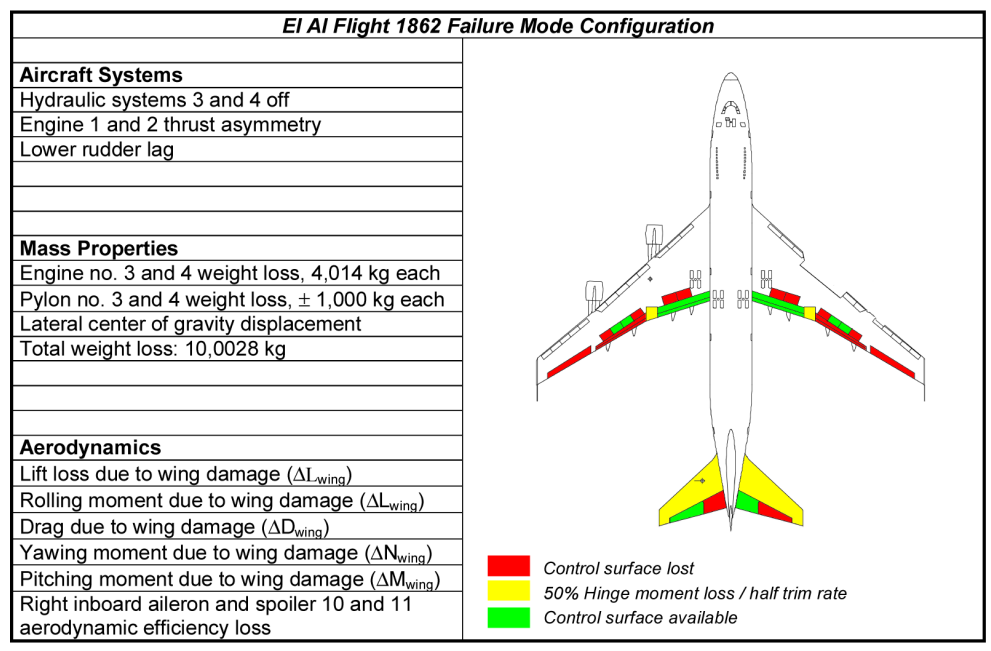

Figure 4: Flight1862 failure effects on the aircraft. ${ }^{20}$

\section{B. Flight Procedure}

The initial properties of the aircraft for the flight scenarios are listed in Table 5 and the flight plans are visualized in Figure 5. The aircraft is exceeding its maximum landing weight of $238,776 \mathrm{~kg}$, because the initial weight of Flight1862 was used as an initial condition. ${ }^{7,21}$ It should be noted that all the flight scenarios were flown without wind, gust and turbulence. A study of Falkena et al. already showed that a SBB controller has sufficient disturbance rejection properties to cope with influences of wind, gust and turbulence. ${ }^{9}$ As the SB-NDI controller is only different with the outer loop structure when compared with an SBB controller to control the attitude rates, it is expected that the designed multi-loop SB-NDI controller will have adequate disturbance rejection properties as long as the aircraft is within safe operating limits.

Table 5: Initial properties of the B747 100/200.

\begin{tabular}{lc}
\hline \hline Property & Value \\
\hline Mass & $317,000 \mathrm{~kg}$ \\
Indicated airspeed & $275 \mathrm{kt}$ \\
Altitude & $2000 \mathrm{ft}$ \\
\hline \hline
\end{tabular}

The flight plan for the nominal and the Flight1862 failure configuration are based on the flight of the 1992 Amsterdam Bijlmermeer disaster and Lombaerts et al.'s piloted evaluation in order to keep a consistent flight plan to compare the flights with past results. ${ }^{21,15}$ The first phase of the nominal and Flight1862 flight scenario is visualized in Figure 5a and consists of several flight phases. The initial flight starts with a short normal flight to let the pilot correct for any deviations from the nominal trimmed flight condition.

This initial flight is followed by a 90 degrees right turn after which the failure is introduced. The pilot is informed when inducing the failure to take away the 'surprise' effect and reduce the amount of time spend in the post-failure identification and decision process. ${ }^{15}$ During this phase, the pilot mainly spends his time identifying the type of failure and formulating a control strategy. By informing the pilot, the failure identification process is taken away and the pilot can direct his attention to controlling the aircraft. It should be explicitly stated that only the pilot was informed of the failure, the controllers do not receive any information regarding changes of the controlled system.

Once the pilot has brought the aircraft in a quasi-stable flight condition, several maneuvers are executed to evaluate the longitudinal and the lateral stability of the post-failure aircraft. ${ }^{15}$ The pilot is requested to bring the aircraft with an acceptable rate of climb to an altitude of $2500 \mathrm{ft}$ to evaluate the longitudinal post-failure flight performance and stability of the controller. During this maneuver, the heading and the 


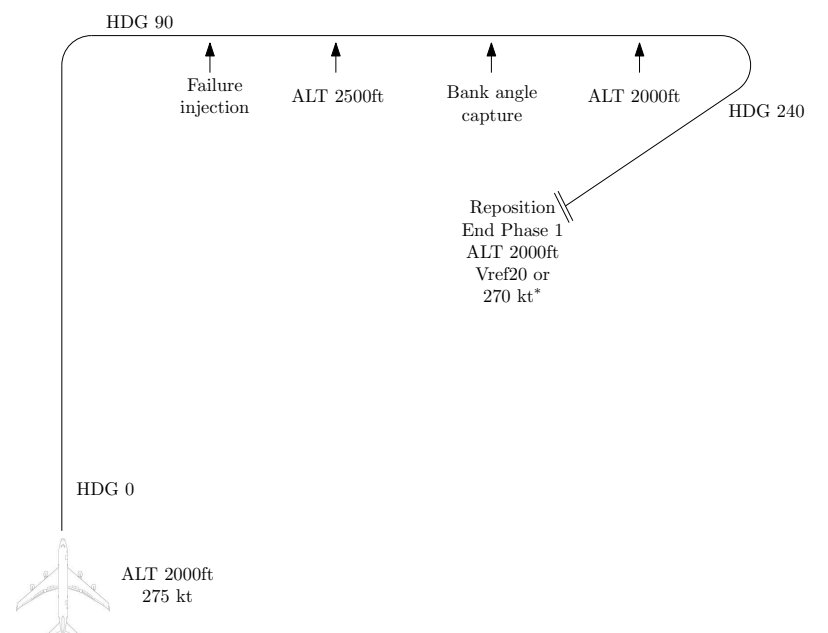

(a) Nominal or engine separation flight route, phase one

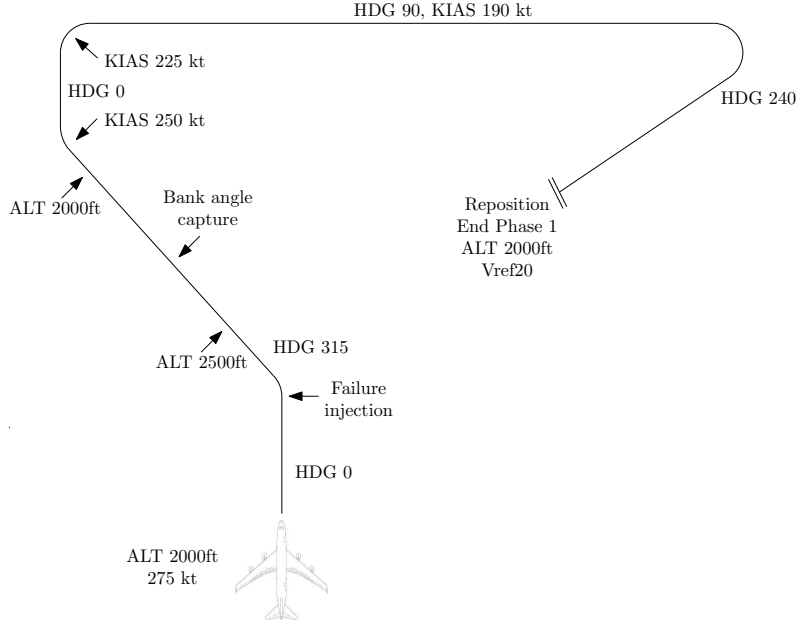

(b) Vertical tail loss flight route, phase one

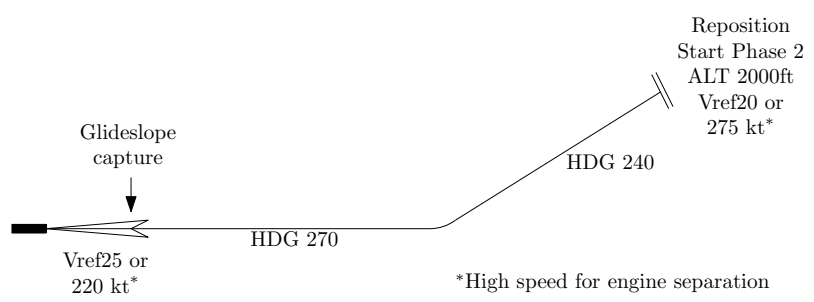

(c) Localizer and glideslope intercept, phase two

Figure 5: Definition of the evaluation flight as was flown in the simulator. ${ }^{15}$

airspeed are kept constant as much as possible. When the aircraft reaches the target altitude, the pilot is subsequently requested to perform an alternating bank angle capture and holding at 20 degrees for two times to evaluate the lateral stability of the controller, while maintaining the same altitude and airspeed.

The bank angle capture is followed by a descent back to an altitude of $2000 \mathrm{ft}$ with the same heading and airspeed. Phase one of this flight plan ends with a right turn to heading 240 to prepare for the Instrument Landing System (ILS) localizer intercept of runway 27 of the airport and the simulation is paused to reposition the aircraft for the beginning of phase two. Conventional approach procedures apply for this turn-in maneuver which means that the pilot is requested to decelerate the aircraft to $174 \mathrm{kt}$ (Vref20 for the current mass of $317,000 \mathrm{~kg}$ ) and to extend the flaps to flaps $20 .^{2}$ However, the engine separation scenario uses a modified procedure and intercepts the localizer with a high velocity profile at a velocity of $275 \mathrm{kt}$ to prevent loss of control and to illustrate that the controller can operate within the safe flight envelope of the post-failure aircaft. ${ }^{21,15}$ The ability of the controllers to stabilize the aircraft at lower speeds, outside of the Flight1862 envelope, remains limited by the aircraft's physical constraints. Furthermore, it should be taken into account that an accurate aerodynamic model for flap and gear configuration changes are not implemented in the RECOVER model for the Flight1862 configuration and thus the flaps are kept constant at flaps 1 with a gear up configuration during this turn-in maneuver. Table 6 summarizes the aircraft configurations for the first phase of the simulation runs. ${ }^{7,15,2}$

Phase one was slightly modified for the vertical tail loss scenario and is shown in Figure 5b. Additional turns with varying flight speeds and a bank angle limit of 20 degrees are introduced to assess the lateral stability properties of the post-failure aircraft. Similar to the nominal and the Flight1862 flight scenario, the vertical tail loss ends with a right turn to heading 240 according to the conventional approach procedures. As a result, the aircraft has an airspeed of $174 \mathrm{kt}$ with flaps 20 for this scenario before repositioning.

The aircraft is repositioned at the end of phase one to keep the final approach phase constant between the controller evaluations for each scenario and flight controller configuration. The final approach procedure is visualized in Figure 5c and is performed according to the normal approach procedures, except for the 
Table 6: Aircraft configurations during the flight scenarios. (* Flight1862 flight configuration $)^{7,15,2}$

\begin{tabular}{lccc}
\hline \hline Flight Phase & Airspeed (kt) & Flaps & Gear \\
\hline Phase one & Varying & Varying / $1^{*, \dagger}$ & up \\
Localizer intercept (Phase two) & $174 / 270^{*}$ & $20 / 1^{*}$ & up \\
Glideslope intercept (Phase two) & $162 / 220^{*}$ & $30 / 1^{*}$ & down/up* \\
\hline \hline
\end{tabular}

$\dagger$ Only after inducing the Flight1862 failure

Flight1862 case. $^{7}$ Phase two ends once the aircraft has reached an altitude of $200 \mathrm{ft}$ during the approach. It should be noted that the landing itself was not part of the approach procedure, because a realistic aerodynamic ground effect model for the (damaged) aircraft is not implemented in the RECOVER model. However, it is believed that if the pilot is capable of bringing the aircraft in a stable condition within the runway boundaries, the landing itself can be successfully performed. ${ }^{7,15}$

The aircraft is repositioned at the same coordinates as Lombaerts et al.'s flight experiment to provide time to re-stabilize the aircraft after resuming the simulation and to make it easier to intercept the localizer of runway 27 with a high airspeed. ${ }^{15}$ The aircraft is repositioned $5 \mathrm{NM}$ along the track from the interception point to give the pilot additional time for re-stabilizing the aircraft after resuming the simulation run. The interception point itself was also moved $5 \mathrm{NM}$ backwards from the usual interception to give the pilot more time for intercepting the localizer. For the nominal and the vertical tail loss scenario, the localizer is intercepted with a velocity of $174 \mathrm{kt}$ with flaps $20 .^{7,2}$ However, the Flight1862 scenario intercepts the localizer with a high velocity profile of $270 \mathrm{kt}$ and flaps 1 to prevent loss of control due to the limited safe flight envelope of the post-failure aircraft. ${ }^{15}$

After capturing and stabilizing the aircraft on the localizer at an altitude of $2000 \mathrm{ft}$, the pilot resumes with the final descent by intercepting the glideslope. The glideslope was captured with a landing configuration of the aircraft (flaps 30, landing gear down), while reducing the velocity to $162 \mathrm{kt}$ (Vref30 for the current mass of $317,000 \mathrm{~kg}$ ) for both the nominal flight and vertical tail loss scenario. ${ }^{2}$ For the Flight1862 scenario, the velocity was reduced to $220 \mathrm{kt}$ with flaps $1 .{ }^{15}$ Any further reduction of the airspeed would result in a loss of go-around capabilities for the engine separation case with flaps $1 .{ }^{21}$ The simulation ends once the aircraft reaches an altitude of $200 \mathrm{ft}$. The aircraft configurations for phase two are summarized in Table 6.7,15,2

Both the nominal and the Flight1862 cases use the nominal SB-NDI attitude rate controller with the conventional control surfaces. Furthermore, the designed SB-NDI controller also contains an option to employ differential thrust to counteract disturbing yawing when the rudders are disabled. This design variant of the SB-NDI controller with differential thrust is explicitly evaluated with the vertical tail loss. As a result, the pilot has to control the airspeed by setting the reference velocity for this scenario. For the full manual control and the NDI attitude rate controller, the pilot is free to apply differential thrust when he finds it necessary. Finally, the pilot was clearly instructed not to use the yaw pedals when flying with either the NDI or the SB-NDI controllers.

\section{Hardware}

The flights were run on the SRS (Figure 6) at Delft University of Technology. The SRS is a six degrees of freedom research flight simulator with a configurable outside visual and flight instrumentation deck. ${ }^{24}$ The RECOVER model with full manual control, NDI and the SB-NDI controller was converted to C-code by using the Simulink Coder . Elements such as the visuals, pilot interface and the simulation hardware were all connected with each other by means of a middleware software layer called the Delft University Environment for Communication and Activation (DUECA). ${ }^{28}$ This layer provides a modular approach in developing the real-time simulation software by hiding and taking care of the synchronization and communication processes between different software modules. 


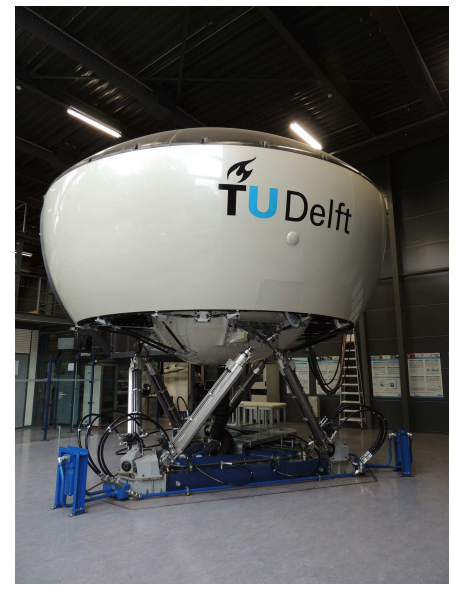

(a) Outside view

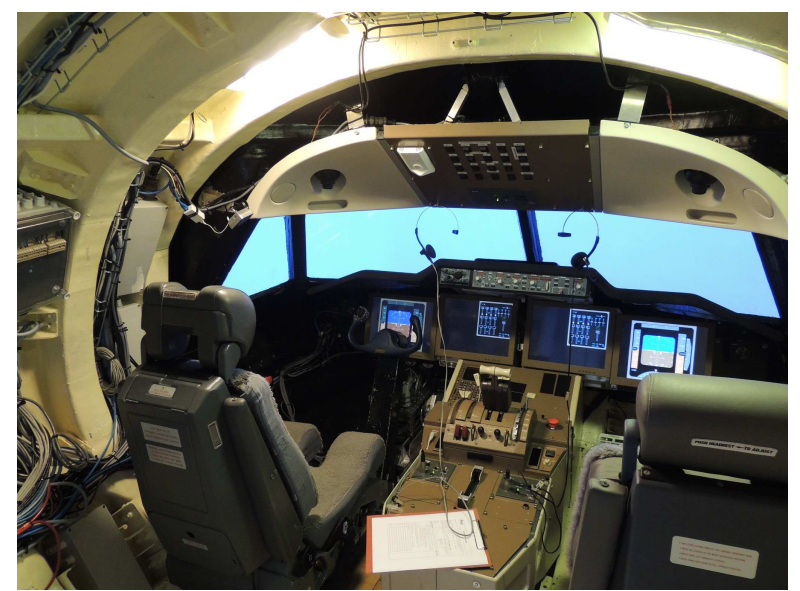

(b) Interior

Figure 6: The SIMONA Research Simulator (SRS).

\section{Computational Hardware}

The dynamics module with the RECOVER model and the flight controllers are run on a desktop computer with a Intel Xeon W3550 @ $3.07 \mathrm{GHz}$. The software model was run single threaded, even though the processor is a quad-core processor.

2. Visuals

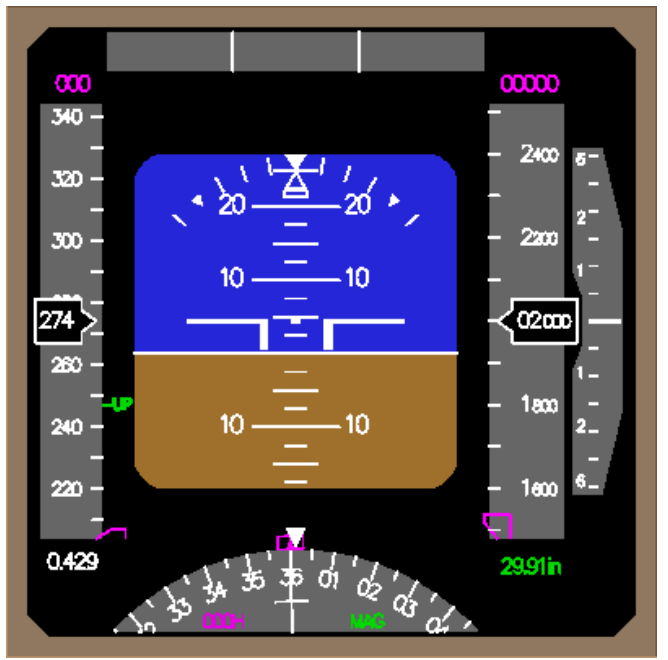

(a) Primary Flight Display

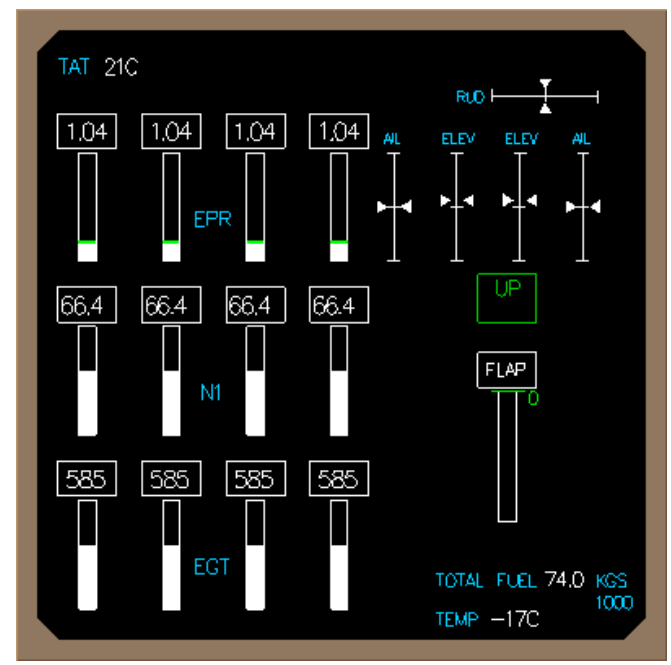

(b) Engine-indication and Crew-alerting System. The AL, ELEV and RUD represent the aileron, elevator and rudder deflections respectively

Figure 7: Used PFD and EICAS interfaces during the simulation run.

The simulation provided the pilots with an outside visual and a flight deck for the instrumentation. The outside visual is provided by FlightGear and consists of a textured map of the Netherlands. This map contains the North Sea and also displays the airport with the major run- and taxiways at their exact location in combination with the most important buildings.

The flight deck of the pilot and the co-pilot consisted of a Primary Flight Display (PFD) interface on the frontal display behind the control column. The Flight Director (FD) was omitted from the PFD which 
meant that the pilot had to land with "raw ILS data." This decision contributed to an increase of the pilot's workload. The pilot was also provided with information about the actuator deflections and the engine status by means of the Engine-indication and Crew-alerting System (EICAS) interface which was displayed on the center screen.

\section{Control Loading Feel System}

Table 7: Characteristics of the control loading system. ${ }^{7,15}$

\begin{tabular}{lcc}
\hline \hline Parameter & Pitch & Roll \\
\hline Arm & $0.714 \mathrm{~m}$ & $0.17 \mathrm{~m}$ \\
Spring constant & $2000 \mathrm{Nm} / \mathrm{rad}$ & $6.93 \mathrm{Nm} / \mathrm{rad}$ \\
Inertia & $5.577 \mathrm{Nm}^{2} / \mathrm{rad}$ & $0.478 \mathrm{Nm}^{2} / \mathrm{rad}$ \\
Damping & $195.3 \mathrm{Nms} / \mathrm{rad}$ & $1.116 \mathrm{Nms} / \mathrm{rad}$ \\
Break-out & $11.1 \mathrm{Nm}$ & $0.1313 \mathrm{Nm}$ \\
Stiction / Friction & $11.1 \mathrm{Nm}$ & $0.1313 \mathrm{Nm}$ \\
\hline \hline
\end{tabular}

The pilot controls the aircraft with a conventional column and pedal system with control loading. The control loading system is modeled as a constant damped mass-spring system and its properties are listed in Table 7.7,15 It should be noted that the control loading does not include a force feedback system from the actuators. The absence of this force feedback system might reduce the situational awareness or the control effectiveness of the pilots, especially in the case of actuator failures.

Because the RECOVER model does not directly support the implementation of aileron and rudder trim inputs, the pilot was supplied with an auto-trim button. This button trims out the applied control forces on the control column and and the pedal once the button is pressed.

\section{Motion System}

The SIMONA Research Simulator (SRS) is a moving base simulator with six degrees of freedom and powered with hydraulics to move the hexapod. The motion filters of SRS can be easily adapted for different flight scenarios or vehicle dynamics. A classical motion filter is used: high-pass filters for all the degrees of freedom, while low-pass filters are used for the tilt coordination of the surge and sway motion. ${ }^{11,17}$ The motion was tuned such that sufficient motion cues were present without hitting the limits of the simulator, while evaluating the flight scenarios.

\section{Physical Workload Analysis}

The physical workload indicates how much physical effort the pilot has to apply in order to successfully complete the requested flight scenario. This paper uses the same approach to analyze the pilot's workload as the work of Lombaerts et al. ${ }^{15}$ The physical workload is analyzed for the full simulation. For scenarios where a failure occurs, the physical workload is analyzed for the time that the failure is induced until the end of the simulation run.

However, the computed values for the workload are not directly comparable, because the recorded data lengths differ for the flights. Therefore, the workload is also analyzed for the localizer intercept phase. This phase is defined as the time span between the valid localizer intercept and the glideslope intercept and makes the computed values for the workload more comparable.

This paper expresses the physical workload by means of the computed Root Mean Square (RMS) value of the applied control deflection and is given by Eq. (22), where $\delta_{c t r l}$ are the applied control deflections on the stick or the control column and $n$ are the amount of data samples. The applied control deflections $\delta_{c t r l}$ are made zero mean to remove the trim inputs of the pilots before computing the RMS values. It should be noted that the analysis does not take the pedal inputs into account, because these were disabled for the SB-NDI and the NDI controller.

$$
R M S_{\delta_{c t r l}}=\frac{\left\|\delta_{c t r l}\right\|_{2}}{\sqrt{n}}
$$




\section{Results}

This section discusses the results obtained by the evaluation flights in the SRS and evaluates the controller performance in a qualitative manner. As a result, the analysis focuses on the most demanding tasks on the pilot after a failure, namely the bank angle captures and the approach procedure. The bank angle capture is defined as the time initiating the bank angle capture until the start of the descent back to the target altitude of $2000 \mathrm{ft}$. The approach procedure is analyzed from the time instance when the localizer is valid until the end of the simulation. In addition to qualitatively evaluating the flight maneuvers, the physical workload is also evaluated according to the methods described in Section IV.

\section{A. Flight Results}

This section describes the time histories of the manually flown flight scenarios with the three different controllers. To compare the actuator deflections, one control surface deflection was chosen from each of the three distinct actuator groups as given by Eq. (8). The representative deflections of each group are the inner left aileron $\delta_{a i l}$ for the ailerons, the inner right elevator $\delta_{\text {eir }}$ for the elevators and the upper rudder $\delta_{r u}$ for the rudder.

\section{Nominal flight}

Figure 8 shows the applied control inputs and the actuator deflections for the controllers during the nominal flight. Figure 8a shows that controlling the roll attitude requires the same magnitude of control deflections, while the pitch inputs differ significantly. Both the SB-NDI or the NDI controller require approximately the same magnitude of pitch inputs for controlling the pitch attitude during the bank angle captures, while flying the aircraft manually requires more and larger inputs.

Despite the reduced control efforts for the nonlinear flight controllers, the pilot did remark that it was harder to fly with the NDI controller due to some longitudinal cross-coupling during the rolling maneuvers. Figure 8 a shows that the pilot needs to apply a pitch input after every roll input when flying with the NDI attitude rate controller. These corrective inputs indicate that there might be indeed a cross-coupling between the lateral and the longitudinal attitude when flying with the NDI controller.

When considering the actuator deflections, Figure $8 \mathrm{~b}$ shows that the resulting aileron deflections are smaller for the SB-NDI and the NDI attitude rate controller when compared with full manual control. This is because the spoilers assist these controllers during a turning maneuver, meaning that less aileron deflection is required to roll. Both the elevators and rudder have the same magnitude of deflections for all the controllers.

Figure 9 indicates that all the controllers have a similar performance during the bank angle capture. The altitude variation for the height $h_{e}$ is within $25 \mathrm{~m}$, while the true airspeed $V_{\text {tas }}$ varies with $5 \mathrm{~m} / \mathrm{s}$. The pitch angle $\theta$ and the angle of attack $\alpha$ remain constant during the bank angle capture, while the heading angle $\psi$ oscillates slightly due to the banking maneuver.

Differences of the flight performance between the controllers are mainly observed for the sideslip angle $\beta$. The outer loop for the SB-NDI and the NDI controller maintains the sideslip angle at 0 degrees, while variations within 2 degrees are observed when flying with manual control.

Figure 10a indicates that more roll input is required for the nonlinear controllers to align with the localizer during the approach phase of the flight when compared with full manual control. However, both the SB-NDI and the NDI require less or even no pitch input at all to maintain the pitch attitude.

Larger differences are observed when considering the actuator deflections which are shown in Figure 10b. The elevator deflections for the SB-NDI and the NDI controller are larger when compared with manual control. This is mainly caused by the fact that these nonlinear controllers use the elevator to maintain aircraft trim during the deceleration, instead of the stabilizer in case of full manual control. Future research could focus on control allocation methods in order to use the other control surfaces more efficiently, while remaining within their operating limits.

The states in Figure 11 show that the approach procedure is the same for all controllers. All controllers follow the same altitude, pitch angle and angle of attack profile, while maintaining the velocity between 

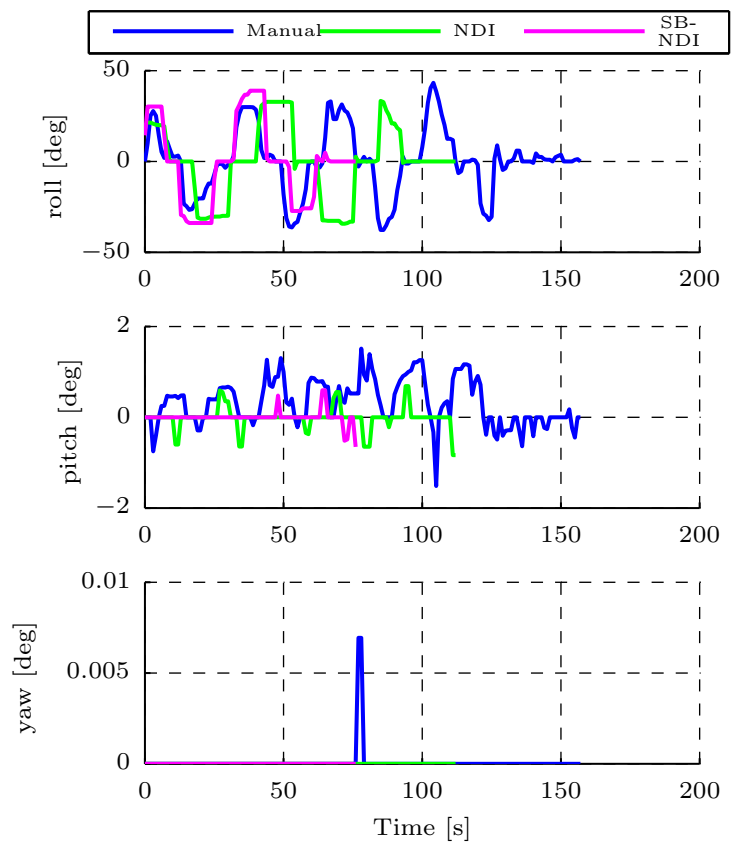

(a) Pilot inputs
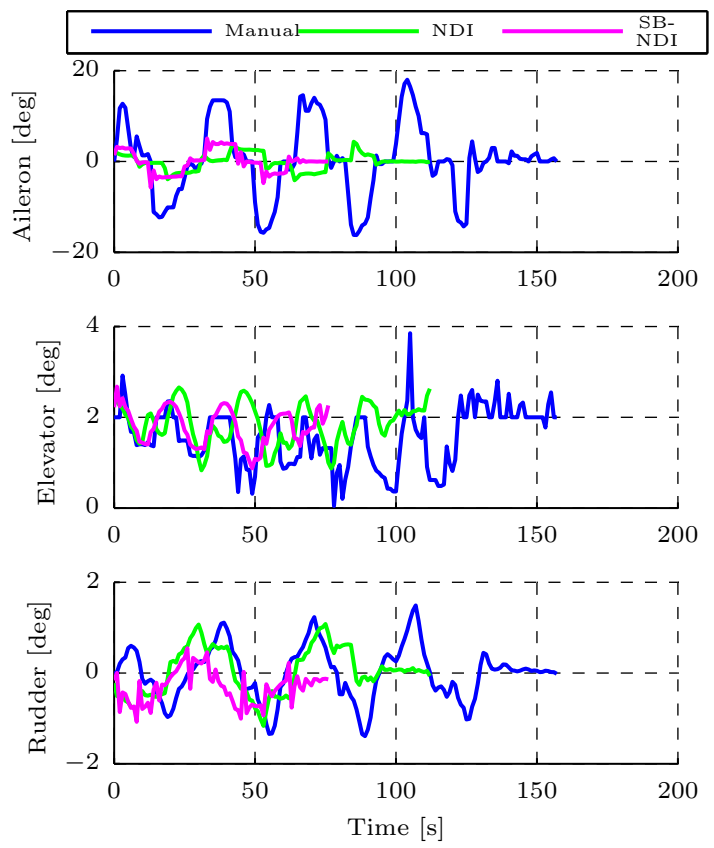

(b) Actuators

Figure 8: Pilot inputs and surface deflections during the bank angle capture for the nominal flight scenario.
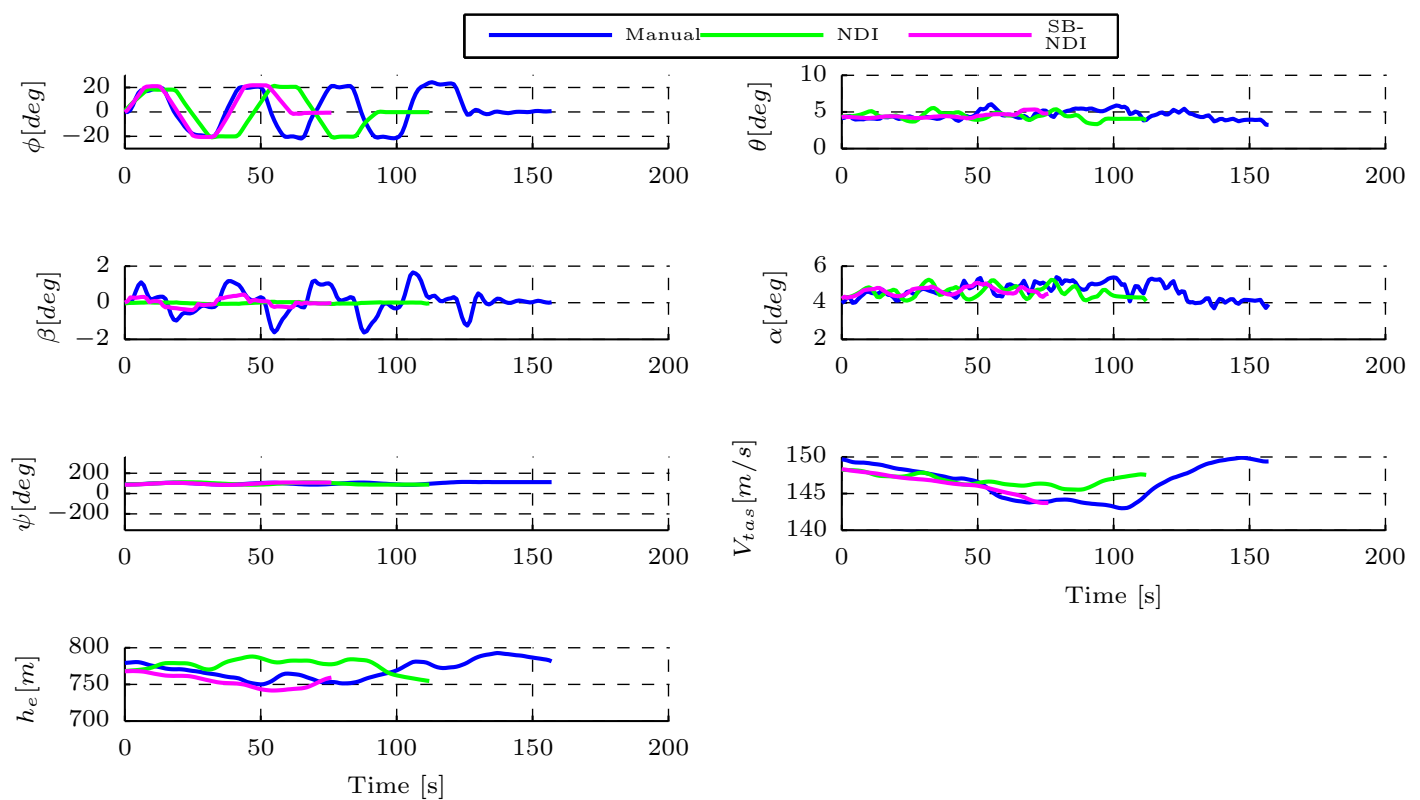

Figure 9: Comparison of the selected aircraft states during the bank angle capture for the nominal flight scenario. 

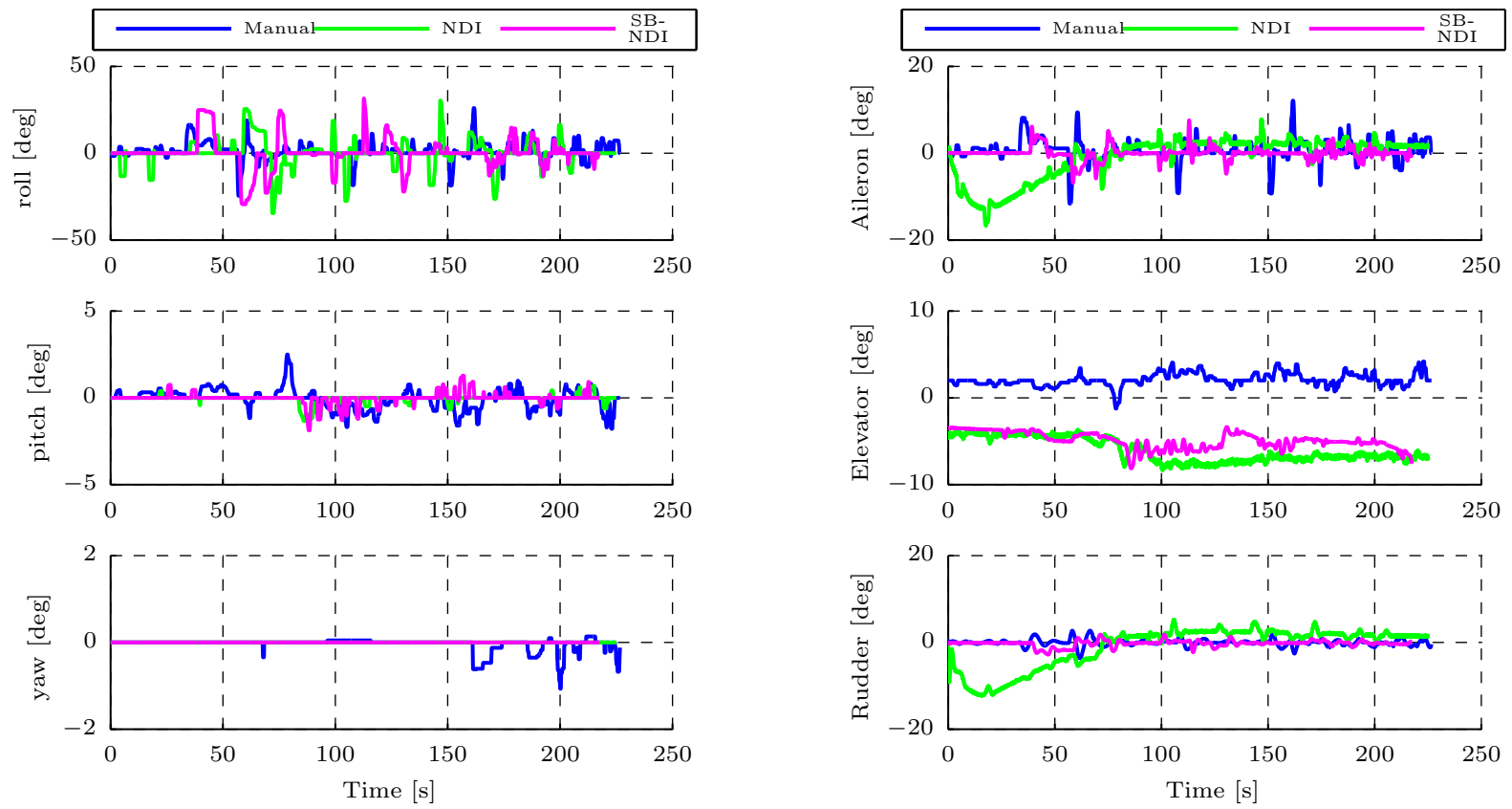

(a) Pilot inputs

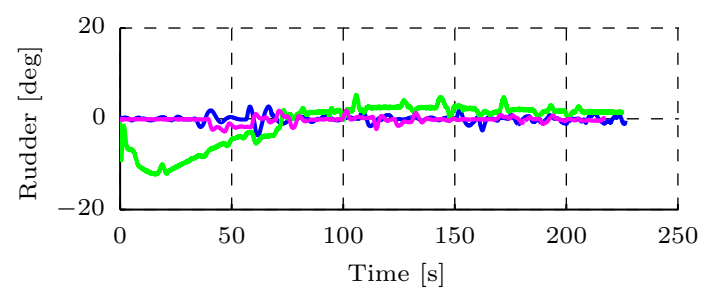

(b) Actuators

Figure 10: Pilot inputs and surface deflections during the approach for the nominal flight scenario.
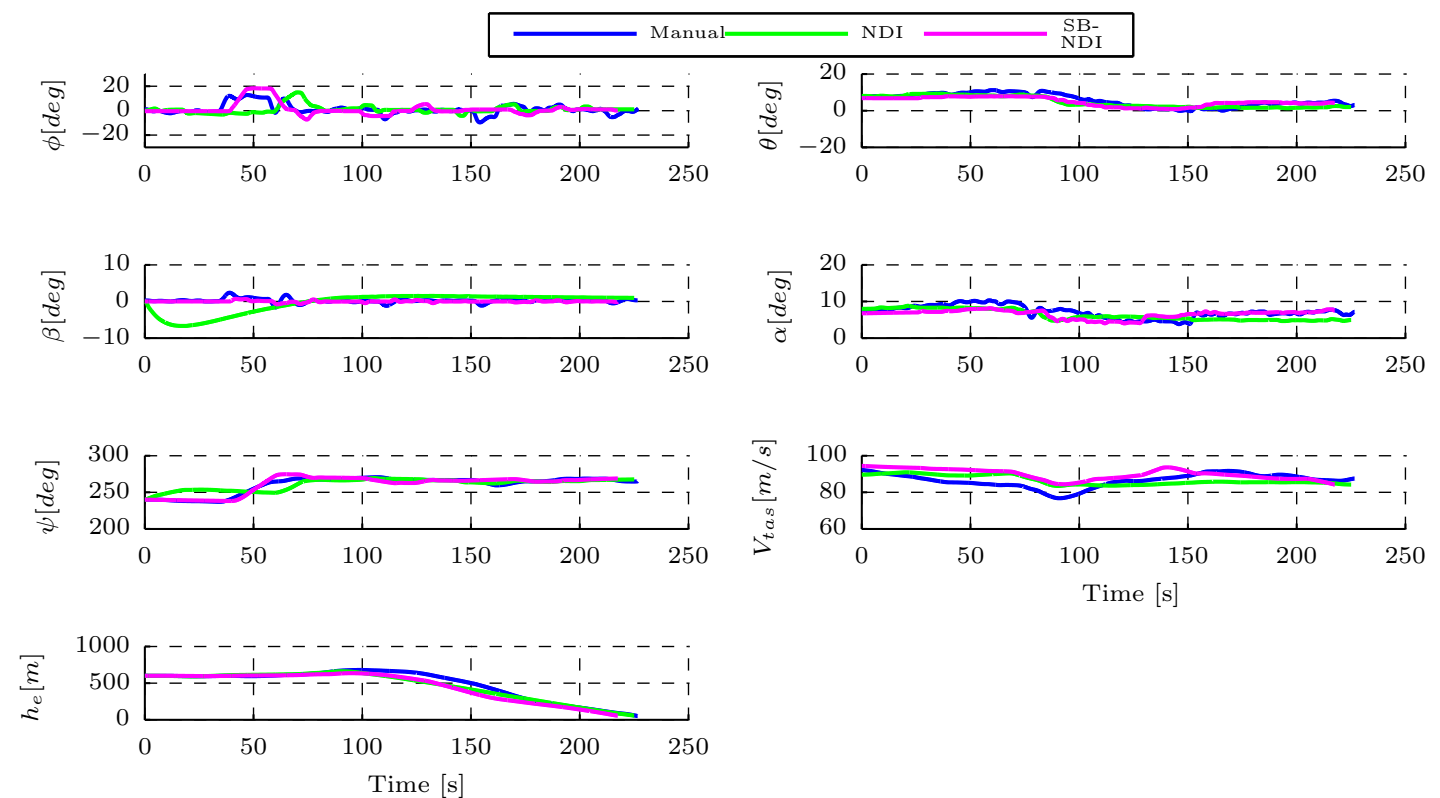

Figure 11: Comparison of the selected aircraft states during the approach for the nominal flight scenario. 
80 and $90 \mathrm{~m} / \mathrm{s}$. However, the roll angle does indicate that the turn-in maneuver to heading 270 was more aggressive for the SB-NDI with a roll angle of 20 degrees when compared with a roll angle of 15 degrees for full manual control and the NDI controller.

Furthermore, the NDI controller displays a sudden large increase of the sideslip angle, up to an angle of 8 degrees at 20 seconds during the approach procedure. As a result, the heading angle goes from the initial heading of 240 degrees to a heading of 250. The actuator deflections in Figure 10b show that after the repositioning the aircraft, the rudder and the aileron deflect for the NDI controller and mainly results in an increase of the sideslip angle. Offline simulations verify that this behavior is caused by an artifact in the simulation software for the NDI controller. Due to inaccurate alignment with heading 240 before repositioning, internal states of the NDI controller mismatch with the simulation model and results in an aileron and a rudder and deflection after repositioning.

\section{Flight1862}

The applied pilot inputs in Figure 12 show that for the bank angle capture with the Flight1862 post-failure aircraft configuration, a larger wheel deflection is required when banking towards the right with the manual controller. Both nonlinear controllers require a similar amount of physical control effort when banking left or right. Similar to the the nominal flight, the pilot needs to continuously apply a pitch input of large magnitude during the maneuver, while the nonlinear SB-NDI and the NDI controllers require small or almost no control input for the pitch channel.

The states in Figure 13 indicate that the controllers have a similar bank angle capture performance for this proof-of-concept evaluation. Differences are mainly observed for the pitch angle and the angle of attack when comparing both nonlinear controllers with full manual control. Both the pitch angle and the angle of attack deviate at most 2 degrees for the nonlinear controllers during the maneuver, while the pitch angle drops 5 degrees when flying with full manual control. As a result, the aircraft descends $100 \mathrm{~m}$ from the target altitude during the first bank angle reversal.

Furthermore, Figure 13 shows that the sideslip angle remains within 2 degrees for all control methods during the bank angle capture. When flying with full manual control the aircraft, a constant pedal input is required as can be seen in Figure 12a. Both the SB-NDI and the NDI controller have a sideslip controller and results in an automatic rudder deflection to compensate the disturbing yawing moments by the engines. The rudder deflection and the zero input for the pedals in Figure 12 verify that both nonlinear controllers automatically deflect the rudder for the Flight1862 flight scenario during the bank angle capture.

The results for the approach phase indicate that more physical effort is required when flying with full manual control in order to safely land the aircraft. Figure 12a shows that the pilot applies a continuous input with a larger magnitude for controlling the aircraft's roll and pitch attitude with respect to the nonlinear controllers. Furthermore, a pedal input is required when flying with full manual control in order to compensate the yawing moment of the engines.

The states in Figure 15 show that the pilot lands with the same descend and deceleration profile when flying manually or with the SB-NDI controller. The main differences of the approach procedure are observed for the roll, heading and the sideslip angle. The sideslip angle is kept close to 0 degrees for the SB-NDI controller due to a sideslip controller, while full manual control displays an oscillatory behavior when trying to keep track of heading 270. Furthermore, minor roll angle variations are observed between the SB-NDI controller and manual control. Not only is the turn-in maneuver from heading 240 to 270 performed more aggressively with 20 degrees by the pilot when flying with the SB-NDI controller, the SB-NDI controller also displays less deviation from 0 degree roll angle when compared with manual control. The reduced amount of variations might be an indication that less corrective actions are required to maintain the heading angle.

Finally, the results show that the pilot was not able to land the aircraft safely with the NDI controller. Despite having enough control authority for the majority of the flight scenario, the NDI flight controller brought the aircraft in an unrecoverable flight condition during the glideslope intercept. When the pilot tried to decelerate the aircraft to $220 \mathrm{kt}$ with the NDI controller, the aircraft started to move outside of the safe flight envelope. Even though the aircraft remained well above the minimum controllable airspeed of 100 $\mathrm{m} / \mathrm{s}$, control was lost during the deceleration. ${ }^{15,21}$ The actuator deflections in Figure $14 \mathrm{~b}$ shows that the rudder suddenly increases its deflection at the time of the deceleration, while the inner left aileron gets fully 

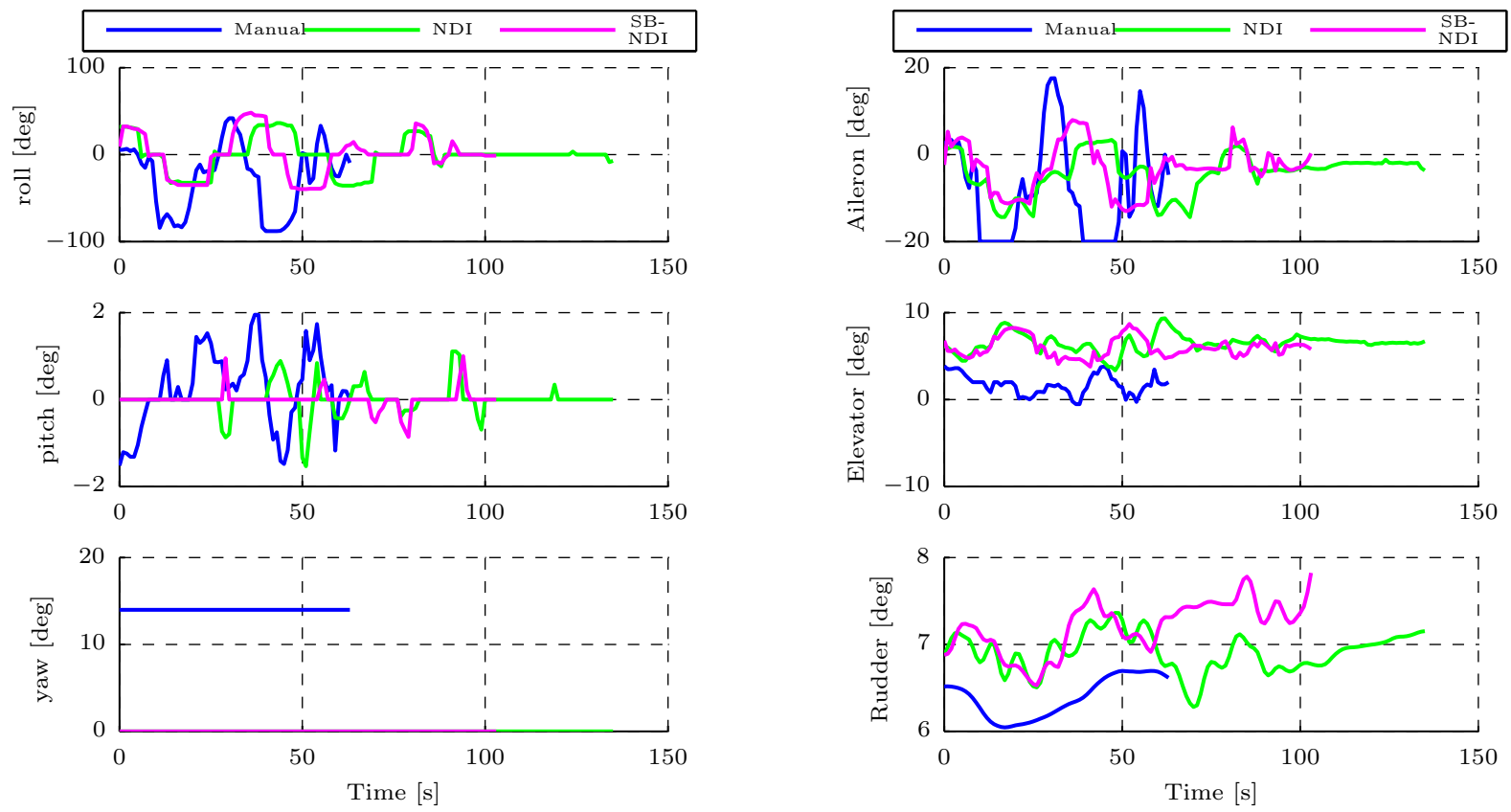

(a) Pilot inputs

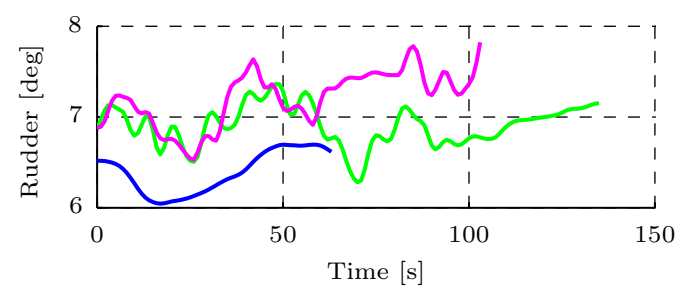

(b) Actuators

Figure 12: Pilot inputs and surface deflections during the bank angle capture for the Flight1862 scenario.
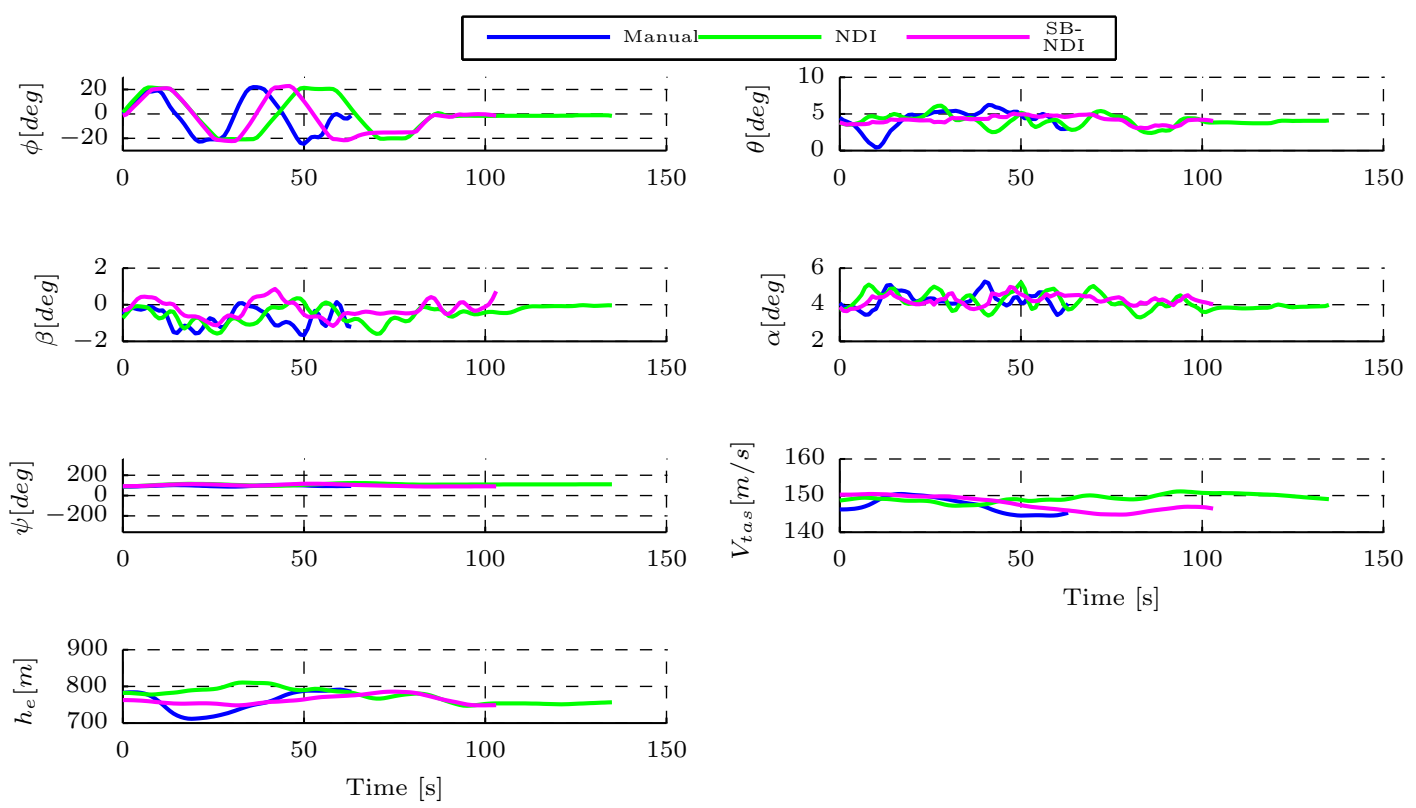

Figure 13: Comparison of the selected aircraft states during the bank angle capture for the Flight1862 scenario. 

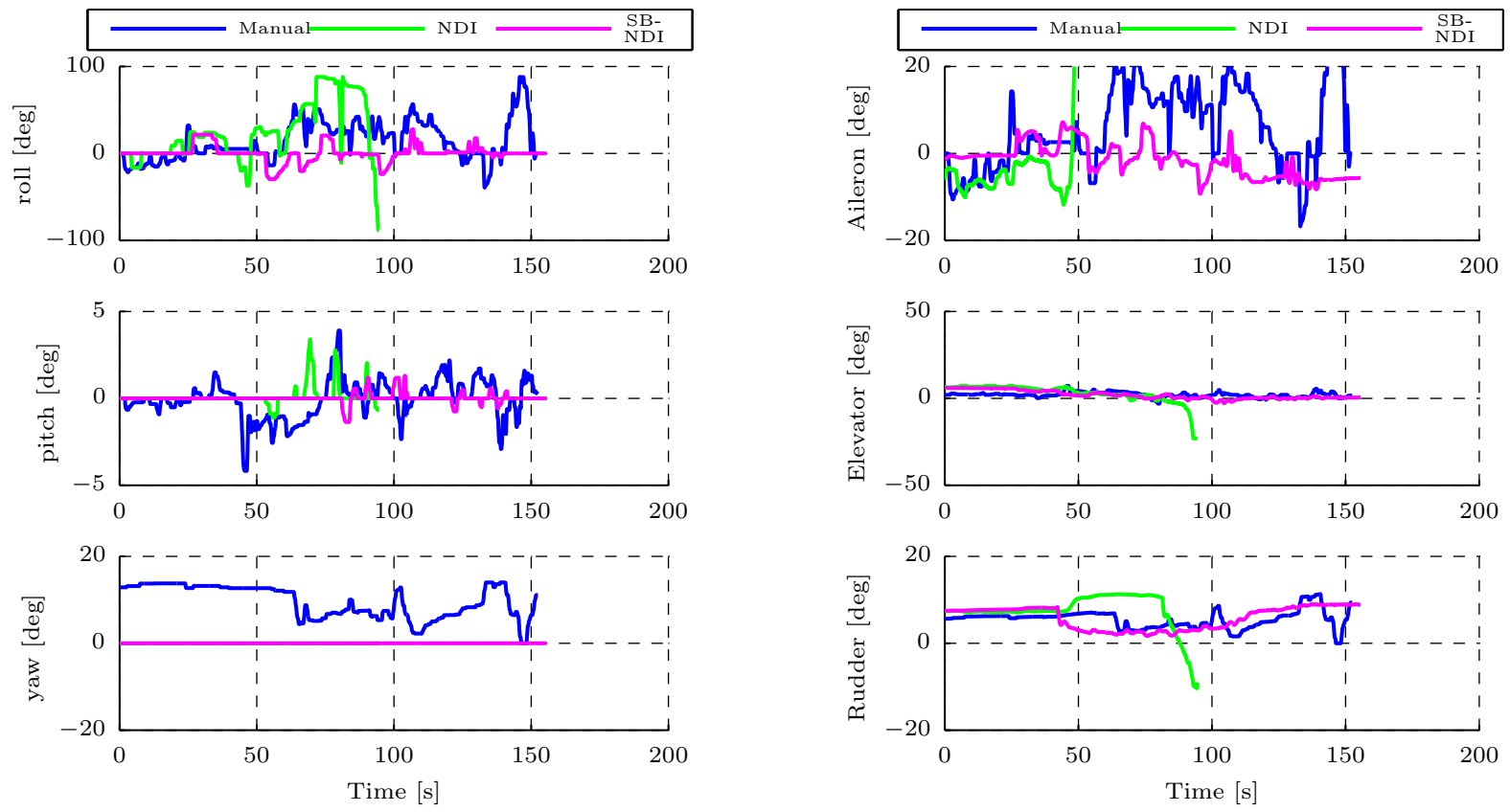

(a) Pilot inputs

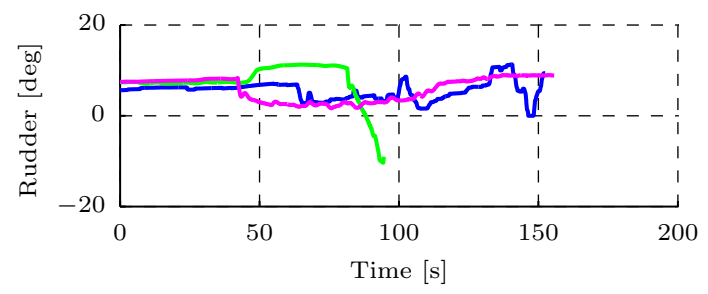

(b) Actuators

Figure 14: Pilot inputs and surface deflections during the approach phase for the Flight1862 scenario.
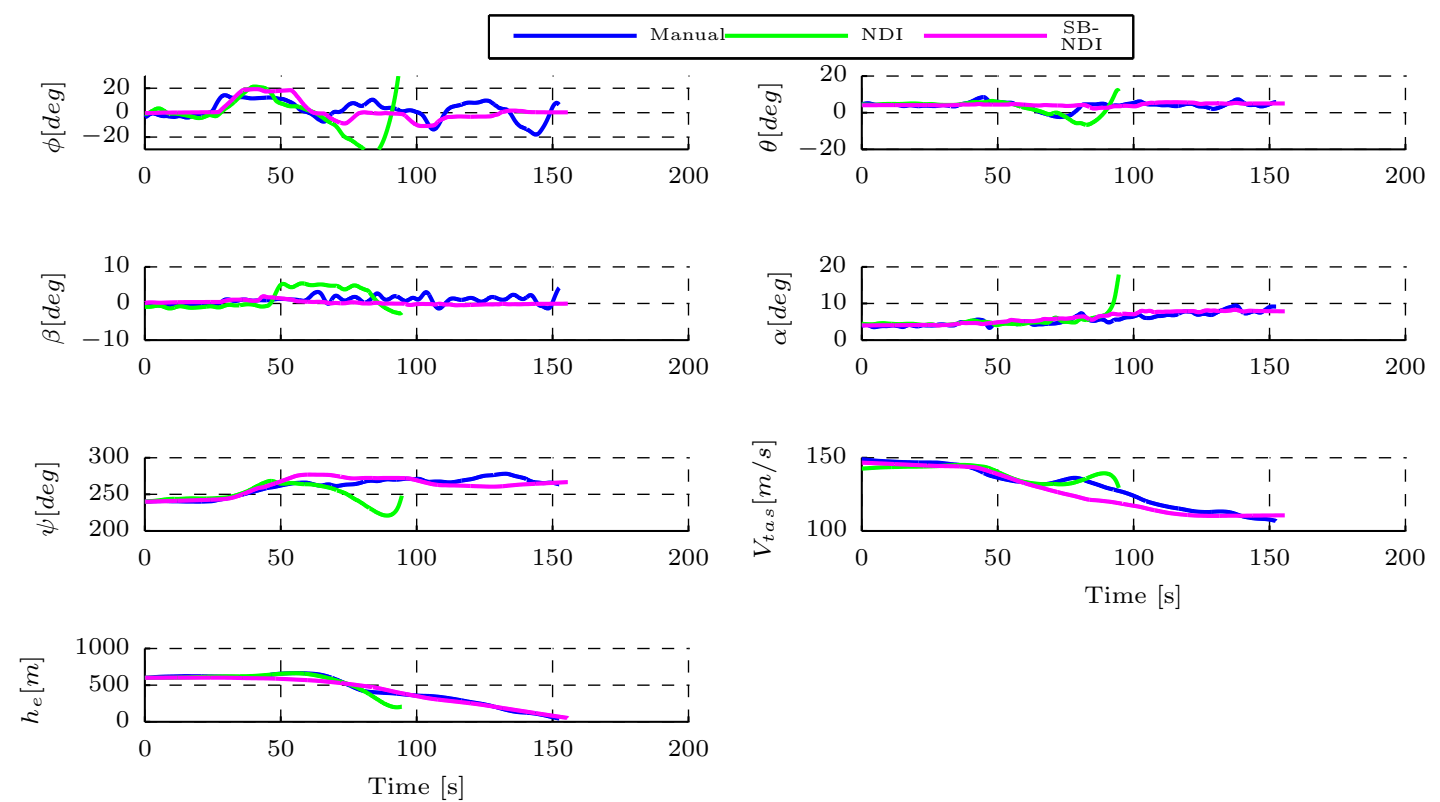

Figure 15: Comparison of the selected aircraft states during the approach phase for the Flight1862 scenario. 
saturated. As a result, the sideslip angle increases in combination with changes in the pitch and roll angle before the aircraft enters an unrecoverable state. Multiple offline simulations could not reveal the cause of why the NDI controller resulted in an unrecoverable situation during this flight scenario and phase.

Nevertheless, this flight scenario does illustrate that the NDI attitude rate controller is robust enough to cope with the additional disturbing rolling and yawing moment disturbances introduced by the Flight1862 flight configuration as illustrated by Figure 4. The Flight1862 flight configuration mainly introduce these disturbing moments in combination with changes in the mass properties of the aircraft and do not significantly change the dynamic stability properties of the aircraft.

\section{Vertical Tail Loss}

For the second considered failure case, Figure 16 shows that the pilot inputs for the SB-NDI controller are comparable when flying with full manual control. Interestingly, the pilot also instinctively uses the pedals when performing the bank angle capture, even though he was informed that the vertical tail was lost. Furthermore, the pilot did not apply differential thrust when flying with full manual control as can be seen in Figure 20.

The selected aircraft states in Figure 17 indicate that the vertical tail loss scenario does not deter the bank angle capture performance when flying with either full manual control or with the SB-NDI controller. Both control methods provide the performance for the bank angle capture as compared with the nominal flight maneuver. During the maneuver, the sideslip angle remains within 5 degrees, while the pitch attitude, airspeed and the altitude are maintained constant.

The flight results also show that the pilot is unable to successfully perform the full flight scenario with the NDI controller. Although the pilot was able to turn to heading 315 with this controller, control was lost as soon as the pilot tried to capture the bank angle for the third time. The pitch angle oscillates extremely, which is likely the cause of an oscillatory elevator deflection as can be seen in Figure 16b. Furthermore, the sideslip angle started to increase to almost 15 degrees before the aircraft enters an unrecoverable state.

The vertical tail loss illustrates that the NDI controller is not robust enough to cope with sign changes of the dimensionless stability derivatives. As the sign of the Weathercock stability derivative $C_{n_{\beta}}$ changes, it is possible that the calculated control actions are inappropriate and causing the aircraft to enter an unrecoverable flight condition, because they are based on a faulty model. The SB-NDI controller does not have this issue, as changes of the aircraft dynamics are indirectly measured by the angular accelerations.

The performance between full manual control and the SB-NDI controller mainly differs when considering the approach phase. The aircraft flies at a lower velocity of $174 \mathrm{kt}$ IAS and results in a more oscillatory response when tracking the heading angle with full manual control. The heading angle oscillates as a result of the oscillating roll and sideslip angle as can be seen in Figure 19. To counteract these effects, the pilot applies more roll input in order to maintain the target heading of the localizer which is shown in Figure 18a. Similar to the bank angle capture, the pilot instinctively uses the pedals to align the aircraft, even though he was informed that the vertical tail was lost. Furthermore, differences are observed for the elevator deflections: this is mainly because the SB-NDI controller uses the elevators to maintain an aircraft trim.

While full manual control requires more pilot inputs on the roll and pitch channels, the SB-NDI controller requires less input from the pilot. In addition, the SB-NDI controller provides a better, less oscillatory tracking performance of the target heading angle. As can be seen in Figure 19, both the sideslip and the heading angle remain fairly constant, while maintaining a constant roll attitude. This performance difference between the SB-NDI controller and full manual control can be explained by the fact that the SB-NDI controller uses differential thrust to compensate the disturbing yawing moments. However, the differential thrust controller also includes a velocity hold mode which results in a noisy auto-throttle input as can be seen in Figure 20. Due to the noisy input, it is not possible to verify the amount of applied differential thrust.

Nevertheless, the pilot commented that flying the vertical tail loss scenario with full manual was manageable at all speeds, as long as the pilot steers calmly. However, he also commented that the bank angle captures and maintaining a constant heading are harder due to the effects of a Dutch roll. 

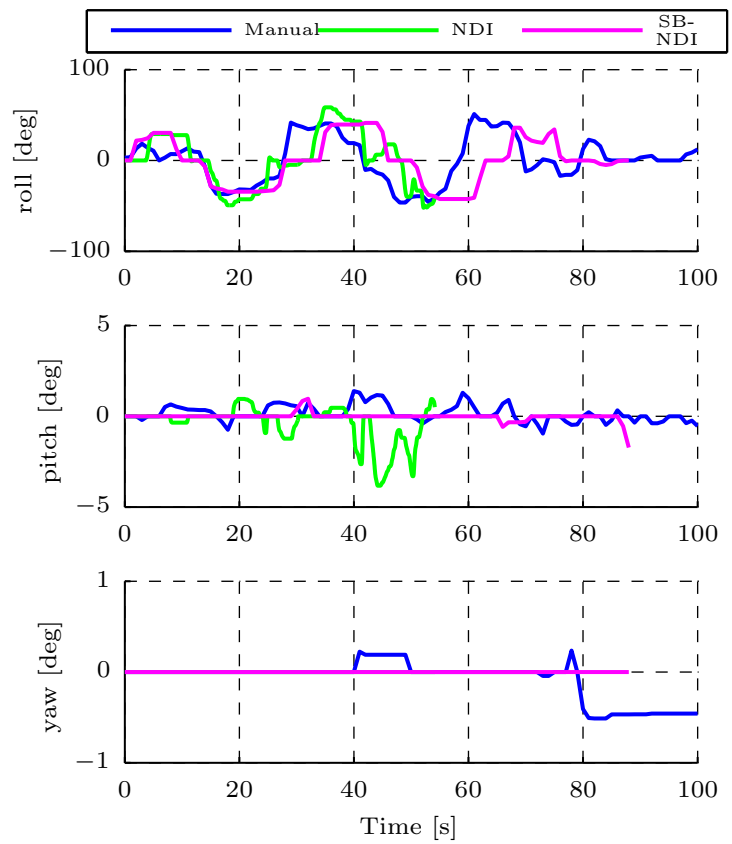

(a) Pilot inputs
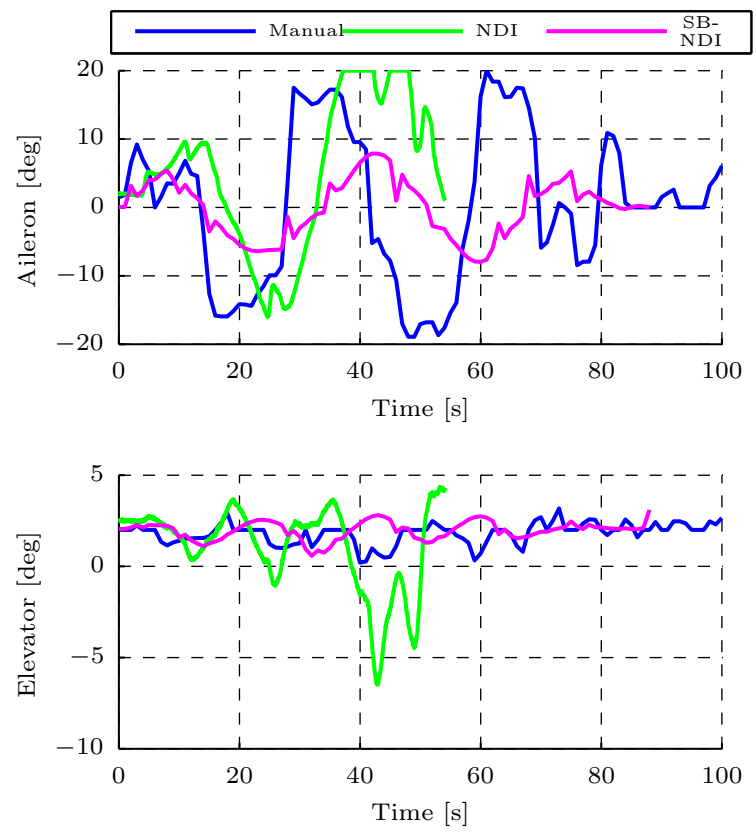

(b) Actuators

Figure 16: Pilot inputs and surface deflections during the bank angle capture for the vertical tail loss scenario.
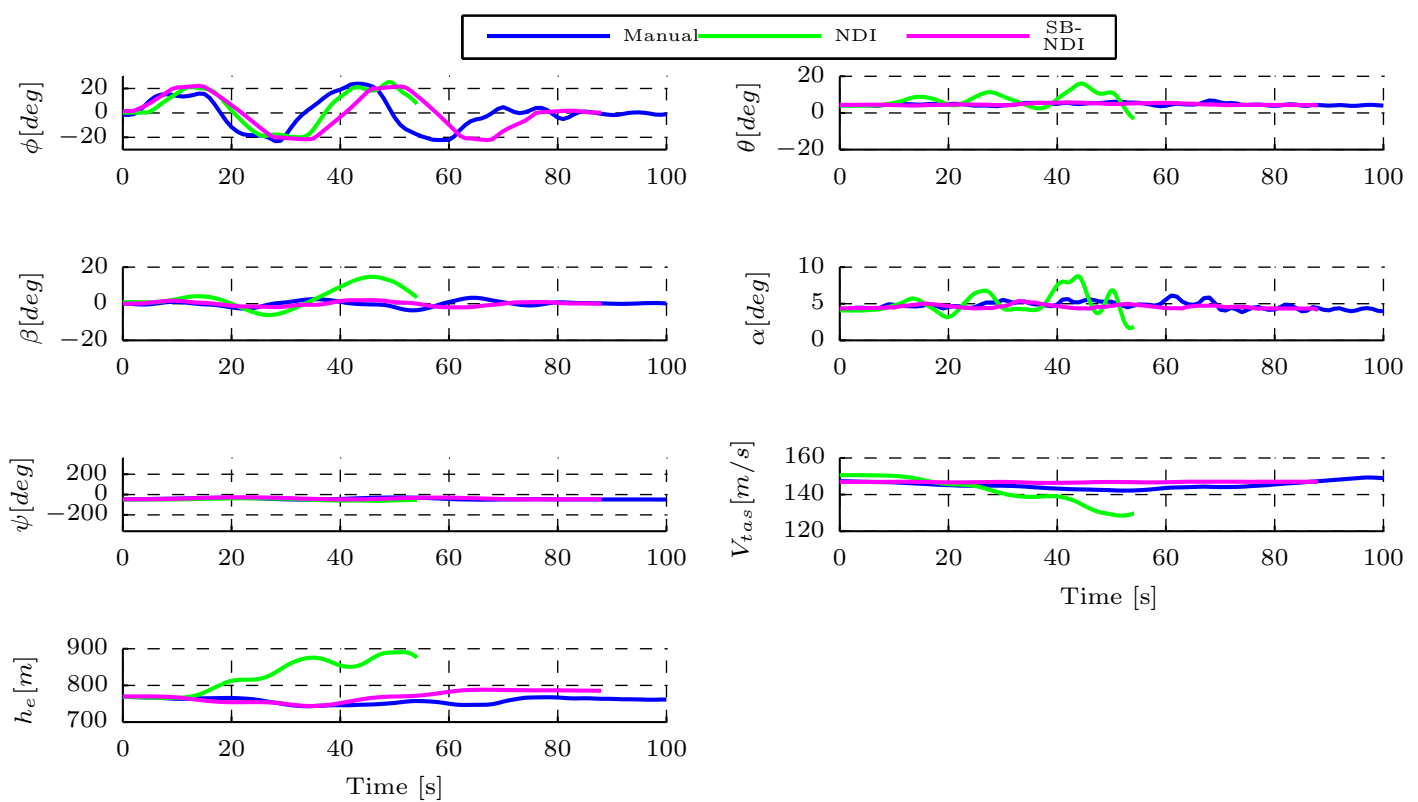

Figure 17: Comparison of the selected aircraft states during the bank angle capture for the vertical tail loss scenario. 

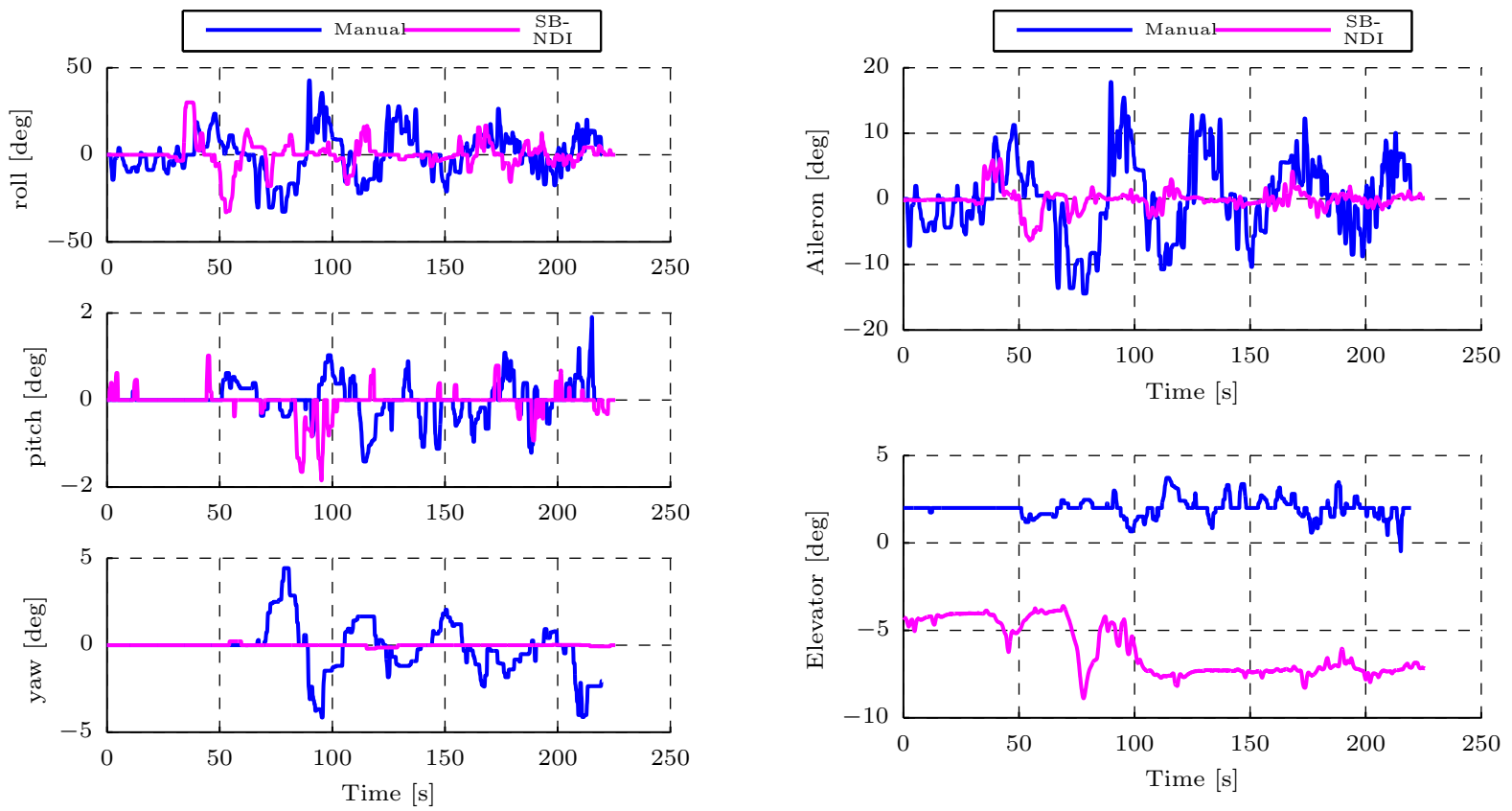

(a) Pilot inputs

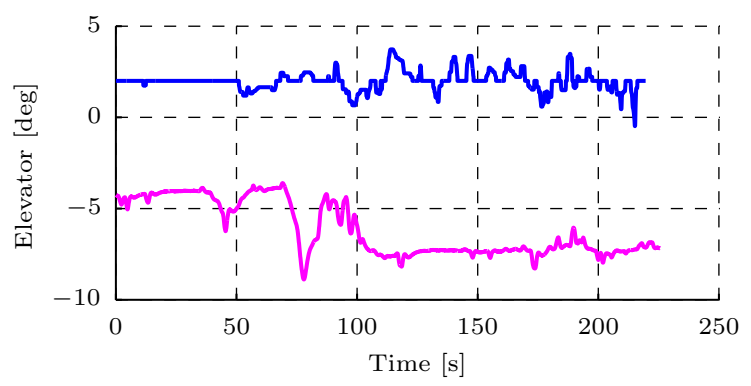

(b) Actuators

Figure 18: Pilot inputs and surface deflections during the approach phase for the vertical tail loss scenario.
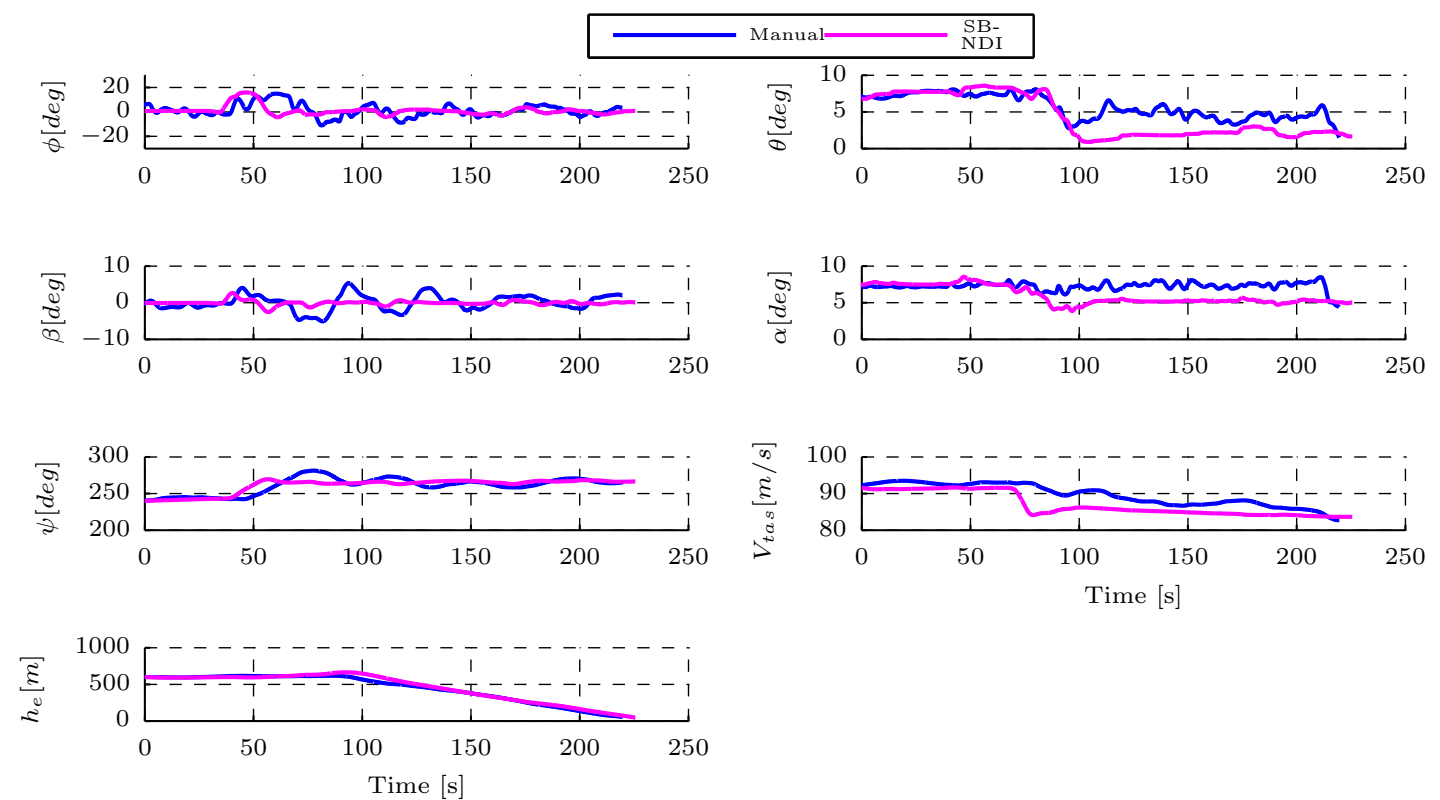

Figure 19: Comparison of the selected aircraft states during the approach phase for the vertical tail loss scenario. 

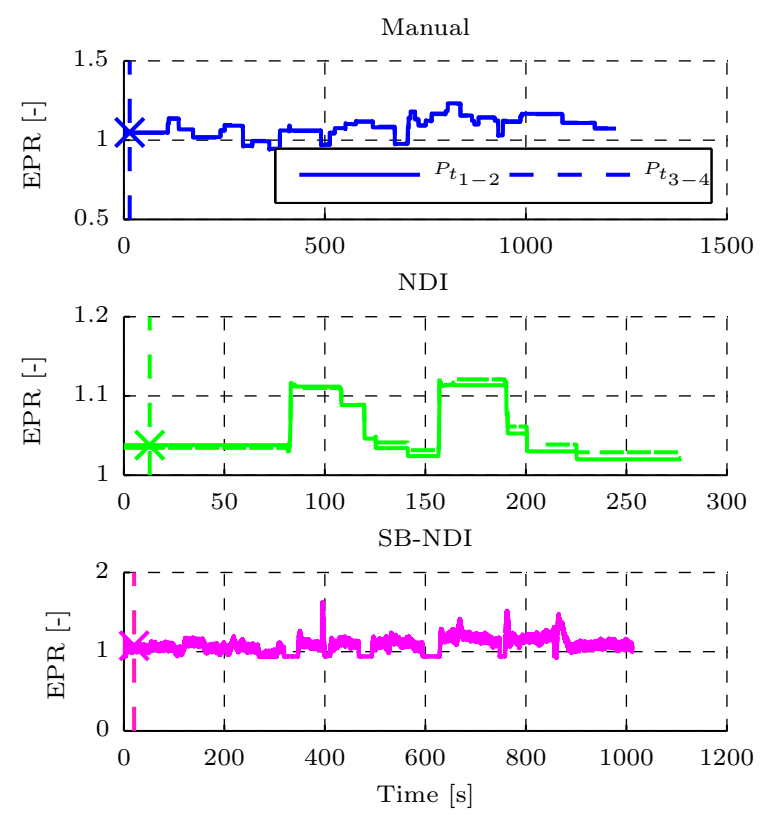

Figure 20: Throttle input during the vertical tail loss scenario. $\times$ indicates the time of inducing the aircraft failure.

\section{B. Physical Workload}

The physical workload was assessed by computing the RMS of the pilot inputs after making the inputs zero mean to remove constant trim inputs. The results are shown in Figure 21. Because the pedal inputs were not used as inputs for the SB-NDI and the NDI controller, the RMS values of only the pitch and roll inputs were calculated. Furthermore, as the length of the simulation varies, the RMS was computed for the full run and for the localizer intercept phase. The latter is defined as the time span between intercepting the localizer and the glideslope.

When there is no failure present, the bar graph indicates that it takes a comparable amount of effort to control the roll attitude with every controller, as the RMS values are of equal magnitude. However, controlling the pitch attitude takes more effort with full manual control. This result is supported by the pilot inputs of the corresponding flight scenario in Figures 8 and 10. Pitch inputs when flying the aircraft with full manual control are of a larger magnitude and rarely remain zero. The same trend is visible for the localizer intercept phase: controlling the roll attitude requires a similar amount of effort regardless of the controller, while the pitch attitude requires more effort when flying with full manual control. Furthermore, the RMS values indicate that controlling the pitch requires less effort with the NDI attitude rate controller when compared with the SB-NDI controller during the localizer intercept phase.

The results for the engine separation scenario show that the physical workload depends on the used controller. Figure 21 shows significantly increased RMS values for the roll and pitch inputs when the pilot flies with full manual control, while the RMS values remain comparable with the nominal flight scenario for the NDI or the SB-NDI controller. However, the RMS value of the NDI controller for the roll input is larger when compared with the SB-NDI controller. This increase can be attributed to the final phase of the flight with the NDI controller. The pilot tries to prevent the aircraft from rolling away during the glideslope intercept phase by applying large wheel inputs and thus resulting in a higher RMS value for the roll input.

During the localizer intercept phase, the RMS values for the SB-NDI and the NDI controller increase slightly for the roll input when compared with the nominal flight scenario. However, this increase is still less than the increase for full manual control, meaning that the test pilot for the flight evaluations requires 


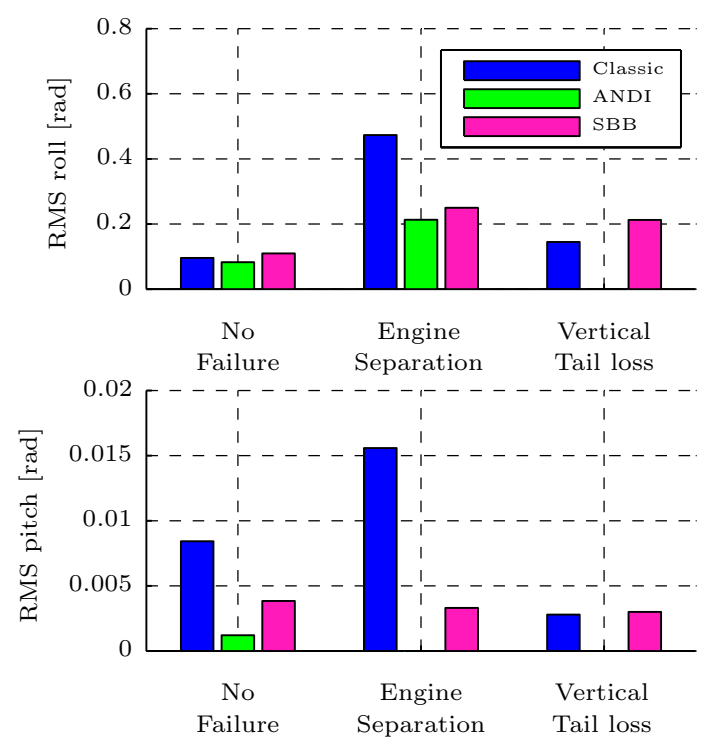

(a) Localizer intercept phase

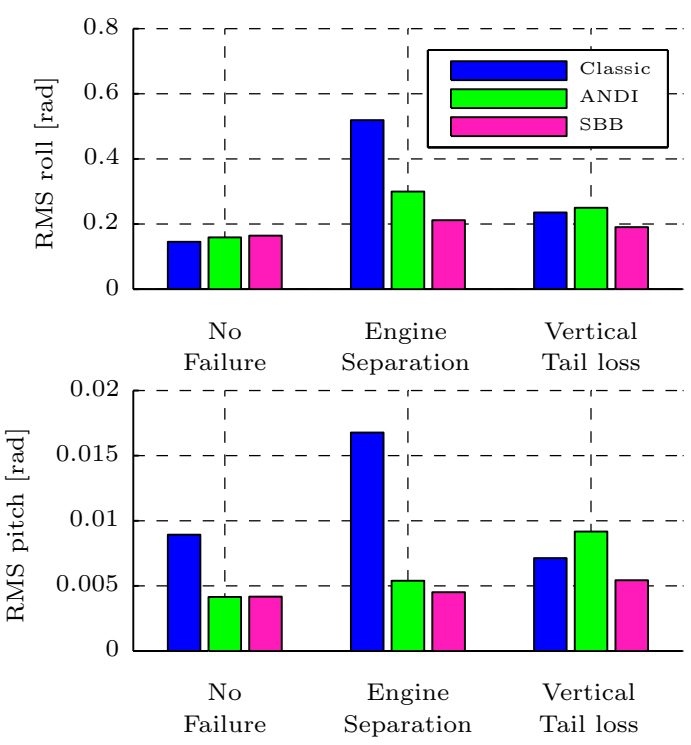

(b) Full run

Figure 21: RMS values of the pilot deflections.

less effort to control the roll attitude of the aircraft with an SB-NDI or an NDI attitude rate controller. Furthermore, the RMS value of the pitch input indicates that no additional effort is required to control the pitch attitude with the SB-NDI or the NDI attitude controllers during this phase. The RMS value for the pitch input is even zero for the NDI attitude controller, because the pilot does not apply any pitch input during this phase.

Considering the vertical tail loss scenario, no increase in the physical workload was observed for full manual control and the SB-NDI controller. The results of Figure 21 indicate that both the manual control and the SB-NDI flight controller require a comparable physical control effort during a vertical tail loss as compared with the nominal flight scenario. RMS values are larger for the NDI controller, especially for the pitch inputs. This increase can be attributed to the attempts of the pilot to (unsuccessfully) bring the aircraft back to stable flight condition.

The localizer intercept phase shows that the RMS values for the roll input are slightly increased for both the manual control and the SB-NDI controller which means that more effort to control the roll attitude is required during the localizer intercept phase. Another observation during this proof-of-concept flight is that the RMS value for the pitch is decreased for both of the controllers during the same phase. A possible explanation for this decrease is the setup of the evaluation. The controllers were evaluated in a fixed sequence with the vertical tail loss scenario as the last flight scenario. It is possible that the decrease of the RMS value for the pitch is caused due to a learning effect. It should also be noted that no data is available for the NDI controller for the localizer intercept phase, because the aircraft entered an unrecoverable flight condition before entering this flight phase.

\section{Conclusion}

This paper presented a proof-of-concept flight evaluation of the fault-tolerant properties of a multiloop Sensor-Based Nonlinear Dynamic Inversion (SB-NDI) attitude rate controller. Three scenarios were considered for this evaluation and were conducted with one test pilot. The multi-loop SB-NDI controller consisted of an inner loop that incorporates the Hovakimyan et al.'s Approximate Dynamic Inversion (ADI) controller in combination with the Nonlinear Dynamic Inversion (NDI) framework and Lyapunov stability to control the angular rates, while the reference angular rates were provided by an NDI-based outer loop. Because the SB-NDI controller is only dependent on the signs of the control effectiveness matrix, the resulting design is fully independent and thus suitable to be implemented as a Fault-Tolerant Flight Controller (FTFC). 
The resulting design was compared with full manual control and an NDI attitude rate controller with a fixed aerodynamic model. The simulations in the SIMONA Research Simulator (SRS) show that designed SB-NDI controller is capable of extending the survivability of an aircraft with a Flight1862 aircraft configuration with a similar bank angle capture performance as an NDI attitude rate controller. Even though simulations with the NDI controller indicate that it is not capable to cope with a vertical tail loss, the NDI controller is robust enough to cope with additional disturbing rolling and yawing moments introduced by the Flight1862 aircraft configuration, because the stability derivatives do not undergo sign changes as a result of the failure.

In terms of control effort, the simulations indicate that both the NDI and the SB-NDI attitude rate controller require a similar amount of physical control effort for a post-failure as an aircraft without failures. This effect is most apparent when flying with the engine separation scenario: the physical workload increases significantly for full manual control, while the NDI and the SB-NDI attitude rate controllers require a comparable physical workload as the nominal flight scenario.

Although this proof-of-concept study shows that the SB-NDI controller successfully extends the survivability of a post-failure aircraft, while requiring a comparable amount of physical control effort as an aircraft without failures, some recommendations for extending this work can be made. Not only should future research focus on conducting flight evaluations with more test subjects to determine the handling qualities, the flight evaluations should also incorporate more flight conditions and failure scenarios in order to assess the robustness of the SB-NDI controller. Besides the experimental setup, the SB-NDI controller itself can be improved by focusing research on control allocation methods to optimally distribute the control actions over the remaining control surfaces. Another improvement for the multi-loop SB-NDI controller is to synthesize a Sensor-Based Backstepping (SBB) attitude rate controller. Instead of using an external NDI loop for the attitude control, the kinematic equations could be incorporated in the final control law and thus resulting in a true Sensor-Based Backstepping attitude rate controller.

\section{Acknowledgments}

The authors would like to thank L. G. Sun for his feedback on the SBB controller design. Furthermore, the authors would like to thank the test pilots J. A. Mulder and A. C. in 't Veld for their participation in the evaluation flights.

\section{References}

${ }^{1}$ Halim Alwi, Christopher Edwards, Olaf Stroosma, and J.a. (Bob) Mulder. Evaluation of a Sliding Mode Fault-Tolerant Controller for the El Al Incident. Journal of Guidance, Control, and Dynamics, 33(3):677-694, May 2010.

${ }^{2}$ Anonymous. Boeing 747 - 400 Flight Crew Operations Manual. 2009.

${ }^{3}$ Anonymous. CAP1036: Global Fatal Accident Review 2002 to 2011. Technical report, CAA-UK, 2011.

${ }^{4}$ Zvr Artstein. Stabilization with relaxed controls. Nonlinear Analysis: Theory, Methods \& Applications, 7(11):1163-1173, 1983.

${ }^{5}$ K. J. Aström and Tore Hägglund. PID controllers: theory, design and tuning. Instrument Society of America, Research Triangle Park, NC, 2nd edition, 1995.

${ }^{6}$ Götz Bramesfeld, Mark D. Maughmer, and Steven M. Willits. Piloting Strategies for Controlling a Transport Aircraft After Vertical-Tail Loss. Journal of Aircraft, 43(1):216-225, January 2006.

${ }^{7}$ Christopher Edwards, Thomas Lombaerts, and Hafid Smaili. Fault Tolerant Flight Control: A Benchmark Challenge. Springer-Verlag, Berlin Heidelberg, 2010.

${ }^{8}$ W. Falkena, C. Borst, E. R. van Oort, and Q. P. Chu. Sensor-Based Backstepping. Journal of Guidance, Control, and Dynamics, 36(2):606-610, March 2013.

${ }^{9}$ Wouter Falkena. Investigation of Practical Flight Control Systems for Small Aircraft. Wöhrmann Print Service, Zutphen, The Netherlands, 2012.

${ }^{10}$ Jay Farrell, Manu Sharma, and Marios Polycarpou. Backstepping-Based Flight Control with Adaptive Function Approximation. Journal of Guidance, Control, and Dynamics, 28(6):1089-1102, November 2005.

${ }^{11}$ Peter R Grant and Lloyd D Reid. Motion Washout Filter Tuning: Rules and Requirements. Journal of Aircraft, 34(2):145-151, March 1997.

${ }^{12} \mathrm{~N}$ Hovakimyan, Eugene Lavretsky, and Amol Sasane. Dynamic Inversion for Nonaffine-in-Control Systems via Time-scale Separation. Part I. Journal of Dynamical and Control Systems, 13(4):451-465, October 2005.

${ }^{13}$ HK Khalil. Nonlinear systems. Prentice Hall, 2002.

${ }^{14} \mathrm{M}$ Krstic, I Kanellakopoulos, and PV Kokotovic. Nonlinear and adaptive control design. John Wiley \& Sons, New York, NY, 1995.

${ }^{15}$ T. J. J. Lombaerts, M. H. Smaili, O. Stroosma, Q. P. Chu, J. A. Mulder, and D. a. Joosten. Piloted Simulator Evaluation 
Results of New Fault-Tolerant Flight Control Algorithm. Journal of Guidance, Control, and Dynamics, 32(6):1747-1765, November 2009.

${ }^{16}$ T.J.J. Lombaerts, H.O. Huisman, Q. P. Chu, Jan A. Mulder, and Diederick. A. Joosten. Nonlinear Reconfiguring Flight Control Based on Online Physical Model Identification. Journal of Guidance, Control, and Dynamics, 32(3):727-748, May 2009.

${ }^{17}$ L. D. Reid and A. M. Nahon. Flight simulation motion-base drive algorithms: part 2. Selecting the system parameters. PhD thesis, University of Toronto Insstitute for Aerospace Studies, 1985.

${ }^{18}$ S. Sieberling, Q P Chu, and J A Mulder. Robust flight control using incremental nonlinear dynamic inversion and angular acceleration prediction. Journal of Guidance, Control, and Dynamics, 33(6):1732-1742, November 2010.

${ }^{19}$ Hafid Smaili, Jan Breeman, Thomas Lombaerts, and Diederick Joosten. RECOVER : A Benchmark for Integrated Fault Tolerant Flight Control Evaluation. pages 171-221, 2010.

${ }^{20} \mathrm{MH}$ Smaili and J Breeman. A simulation benchmark for integrated fault tolerant flight control evaluation. In AIAA Modeling and Simulation Technologies Conference and Exhibit, number AIAA 2006-6471, Keystone, Colorado, August 2006. American Institute of Aeronautics and Astronautics (AIAA)

${ }^{21} \mathrm{MH}$ Smaili and JA Mulder. Flight data reconstruction and simulation of the 1992 Amsterdam Bijlmermeer airplane accident. In AIAA Modeling and Simulation Technologies Conference and Exhibit, number AIAA-2000-4586, Denver, Colorado, August 2000. American Institute of Aeronautics and Astronautics (AIAA).

${ }^{22}$ Vahram Stepanyan, Nhan Nguyen, and Kalmanje Krishnakumar. Adaptive Control of a Transport Aircraft Using Differential Thrust. In AIAA Guidance, Navigation, and Control Conference, number AIAA 2009-5741, Chicago, Illinois, August 2009. American Institute of Aeronautics and Astronautics (AIAA).

${ }^{23}$ B.L Stevens and FL Lewis. Aircraft control and simulation. John Wiley \& Sons, 1992.

${ }^{24}$ Olaf Stroosma, M.M. (René) van Paassen, and Max Mulder. Using the SIMONA Research Simulator for Human-machine Interaction Research. AIAA Modeling and Simulation Technologies Conference and Exhibit, (AIAA 2003-5525), August 2003.

${ }^{25}$ L. G. Sun, C. C. de Visser, Q. P. Chu, and W. Falkena. Hybrid Sensor-Based Backstepping Control Approach with Its Application to Fault-Tolerant Flight Control. Journal of Guidance, Control, and Dynamics, 37(1):59-71, January 2014.

${ }^{26}$ Liguo. G. Sun, Coen C. de Visser, Wouter Falkena, and Q Ping Chu. A Joint Sensor Based Backstepping Approach For Fault-Tolerant Flight Control of a Large Civil Aircraft. In AIAA Guidance, Navigation, and Control (GNC) Conference, number AIAA 2013-4528, Boston, MA, August 2013. American Institute of Aeronautics and Astronautics (AIAA).

${ }^{27}$ H. J. Tol, C. C. de Visser, E. van Kampen, and Q. P. Chu. Nonlinear Multivariate Spline-Based Control Allocation for High-Performance Aircraft. Journal of Guidance, Control, and Dynamics, 37(6):1840-1862, November 2014.

${ }^{28}$ M.M. van Paassen, O. Stroosma, and J. Delatour. DUECA — Data-driven activation in distributed real-time computation. In AIAA Modeling and Simulation Technologies Conference and Exhibit, number AIAA-2000-4503, Denver, Colorado, August 2000. American Institute of Aeronautics and Astronautics (AIAA).

${ }^{29}$ A Visioli. Modified anti-windup scheme for PID controllers. IEEE Proceedings-Control Theory and Applications, pages $49-54,2003$. 